\title{
CSI 2264: Probing the inner disks of AA Tauri-like systems in NGC 2264 ${ }^{\star}, \star \star$
}

\author{
P. T. McGinnis ${ }^{1}$, S. H. P. Alencar ${ }^{1}$, M. M. Guimarães ${ }^{2}$, A. P. Sousa ${ }^{1}$, J. Stauffer ${ }^{3}$, J. Bouvier ${ }^{4,5}$, L. Rebull ${ }^{3}$, \\ N. N. J. Fonseca ${ }^{1,4}$, L. Venuti ${ }^{4}$, L. Hillenbrand ${ }^{6}$, A. M. Cody ${ }^{3}$, P. S. Teixeira ${ }^{7}$, S. Aigrain ${ }^{8}$, F. Favata ${ }^{9}$, G. Fưrész ${ }^{10}$, \\ F. J. Vrba ${ }^{11}$, E. Flaccomio ${ }^{12}$, N. J. Turner ${ }^{13}$, J. F. Gameiro ${ }^{14}$, C. Dougados ${ }^{4}$, W. Herbst ${ }^{15}$, \\ M. Morales-Calderón ${ }^{16}$, and G. Micela ${ }^{12}$ \\ 1 Departamento de Física - ICEx - UFMG, Av. Antônio Carlos, 6627, 30270-901 Belo Horizonte, MG, Brazil \\ e-mail: pauline@fisica.ufmg.br \\ 2 Departamento de Física e Matemática - UFSJ - Rodovia MG 443, KM 7, 36420-000, Ouro Branco, MG, Brazil \\ 3 Spitzer Science Center, California Institute of Technology, Pasadena, CA 91125, USA \\ 4 Univ. Grenoble Alpes, IPAG, 38000 Grenoble, France \\ 5 CNRS, IPAG, 38000 Grenoble, France \\ 6 Astronomy Department, California Institute of Technology, Pasadena, CA 91125, USA \\ 7 University of Vienna, Department of Astrophysics, Türkenschanzstr. 17, 1180 Vienna, Austria \\ 8 Department of Astrophysics, Denys Wilkinson Building, University of Oxford, Oxford OX1 3RH, UK \\ 9 European Space Agency, 8-10 rue Mario Nikis, 75738 Paris, Cedex 15, France \\ 10 MIT Kavli Institute for Astrophysics and Space Research, 77 Mass Ave 37-582f, Cambridge, MA 02139, USA \\ 11 U.S. Naval Observatory, Flagstaff Station, 10391 West Naval Observatory Road, Flagstaff, AZ 86001, USA \\ 12 INAF - Observatorio Astronomico di Palermo, Piazza del Parlamento 1, 90134 Palermo, Italy \\ 13 Jet Propulsion Laboratory, California Institute of Technology, Pasadena, CA 91109, USA \\ 14 Instituto de Astrofísica e Ciências Espaciais and Faculdade de Ciências, Universidade do Porto, Rua das Estrelas, \\ 4150-762 Porto, Portugal \\ 15 Astronomy Department, Wesleyan University, Middletown, CT 06459, USA \\ 16 Centro de Astrobiología, Departamento de Astrofísica, INTA-CSIC, PO BOX 78, ESAC Campus, 28691 Villanueva de la Cañada, \\ Madrid, Spain
}

Received 5 December 2014 / Accepted 6 February 2015

\section{ABSTRACT}

\begin{abstract}
Context. The classical T Tauri star (CTTS) AA Tau has presented photometric variability that was attributed to an inner disk warp, caused by the interaction between the inner disk and an inclined magnetosphere. Previous studies of the young cluster NGC 2264 have shown that similar photometric behavior is common among CTTS.

Aims. The goal of this work is to investigate the main causes of the observed photometric variability of CTTS in NGC 2264 that present AA Tau-like light curves, and verify if an inner disk warp could be responsible for their observed variability.

Methods. In order to understand the mechanism causing these stars' photometric behavior, we investigate veiling variability in their spectra and $u-r$ color variations and estimate parameters of the inner disk warp using an occultation model proposed for AA Tau. We also compare infrared Spitzer IRAC and optical CoRoT light curves to analyze the dust responsible for the occultations.

Results. AA Tau-like variability proved to be transient on a timescale of a few years. We ascribe this variability to stable accretion regimes and aperiodic variability to unstable accretion regimes and show that a transition, and even coexistence, between the two is common. We find evidence of hot spots associated with occultations, indicating that the occulting structures could be located at the base of accretion columns. We find average values of warp maximum height of 0.23 times its radial location, consistent with AA Tau, with variations of on average $11 \%$ between rotation cycles. We also show that extinction laws in the inner disk indicate the presence of grains larger than interstellar grains.

Conclusions. The inner disk warp scenario is consistent with observations for all but one star with AA Tau-like variability in our sample. AA Tau-like systems are fairly common, comprising $14 \%$ of CTTS observed in NGC 2264, though this number increases to $35 \%$ among systems of mass $0.7 M_{\odot} \lessgtr M \lessgtr 2.0 M_{\odot}$. Assuming random inclinations, we estimate that nearly all systems in this mass range likely possess an inner disk warp. We attribute this to a possible change in magnetic field configurations among stars of lower mass.
\end{abstract}

Key words. accretion, accretion disks - stars: pre-main sequence - techniques: photometric - techniques: spectroscopic

\footnotetext{
* Based on data from the Spitzer and CoRoT missions, as well as the Canada France Hawaii Telescope (CFHT) MegaCam CCD, the European Southern Observatory (ESO) Very Large Telescope, and the US Naval Observatory. The CoRoT space mission was developed and operated by the French space agency CNES, with participation of ESA's RSSD and Science Programmes, Austria, Belgium, Brazil, Germany, and Spain. MegaCam is a joint project of CFHT and CEA/DAPNIA, at
}

the Canada-France-Hawaii Telescope (CFHT), operated by the National Research Council (NRC) of Canada, the Institut National des Sciences de l'Univers of the Centre National de la Recherche Scientifique (CNRS) of France, and the University of Hawaii.

$\star \star$ Figures 21-24 are available in electronic form at http://www . aanda.org 


\section{Introduction}

Magnetospheric accretion models account for most of the observed characteristics of classical T Tauri stars (CTTS), such as their strong ultraviolet (UV) and optical excess, as well as broad emission lines and forbidden emission lines (Shu et al. 1994; Hartmann et al. 1994; Muzerolle et al. 2001; Kurosawa et al. 2006; Lima et al. 2010). These stars have also been observed to have strong magnetic fields (Johns-Krull et al. 2001) and X-ray emission (e.g., Preibisch et al. 2005). Magnetohydrodynamics (MHD) models suggest that the stellar magnetosphere truncates the inner circumstellar disk at a distance of a few stellar radii, where the disk's matter stress equals the magnetic stress, forcing the material within this truncation radius to be accreted onto the star, following the magnetic field lines (Bessolaz et al. 2008). This forms what are called accretion columns, which are responsible for the broad emission lines observed, particularly those of the hydrogen Balmer series. When the free-falling material reaches the stellar surface, accretion shocks result in hot spots near the magnetic poles of the stars, causing the observed UV and optical excess. Part of the material from the disk can also be ejected in a disk wind, resulting in forbidden emission lines. These stars also present a strong infrared (IR) excess with regard to the photospheric flux because of the absorption and reemission at longer wavelengths of stellar radiation by the circumstellar disk.

Magnetohydrodynamics models (e.g., Kulkarni \& Romanova 2009; Romanova et al. 2009, 2013; Kurosawa \& Romanova 2013) have proposed that CTTS can accrete matter from their circumstellar disks through a stable or an unstable regime. In the stable regime, a misalignment between the star's magnetic field and the star's rotation axis causes the material present in the region near the disk's truncation radius to fall onto the star via two main accretion columns, following the magnetic field lines connecting the disk to the star (Fig. 1). This interaction may also lift dust above the disk midplane, creating an optically thick warp in the inner disk region near the truncation radius, located at the base of these accretion columns. At the other end of each accretion column, an accretion shock on the stellar surface results in a hot spot, located at a high latitude. Observations of the magnetic fields of CTTS have shown that this misalignment between the star's magnetic field and the star's rotation axis is common (Gregory et al. 2012).

Magnetohydrodynamics models also predict that the stellar magnetic field interacts with the disk at and near the co-rotation radius. Differential rotation between the star and the disk, and throughout the accretion columns, results in a distortion of the magnetic field lines, causing them to inflate and eventually disconnect and reconnect, restoring the original field configuration (Zanni \& Ferreira 2013).

Accretion through an unstable regime occurs when Rayleigh-Taylor instabilities between an accretion disk and a stellar magnetosphere cause matter to accrete onto the star via streams or tongues in random locations around the star, causing stochastic photometric variability (see Fig. 1 in Kurosawa \& Romanova 2013).

If a star undergoing stable accretion is seen at a medium or low inclination, its light curve should show periodic variability due to the modulation of its main hot spot. If it is seen at a high inclination (yet not so high that the flared outer disk completely occults the star), the inner disk warp will occult the stellar photosphere periodically, causing flux dips in the star's light curve. If a star undergoing unstable accretion is seen at a medium or low inclination, its light curve will show stochastic variability



Fig. 1. When there is a misalignment between the axis of the (predominately dipolar) magnetic field $(\mu)$ and the stellar rotation axis $(\Omega)$, magnetospheric accretion may occur via the stable accretion mechanism, where two main accretion columns form at the regions where the stellar magnetic pole is closest to the disk. Two main hot spots due to accretion shocks are located near the magnetic poles.

due to the many hot spots on the stellar surface (Stauffer et al. 2014). These hot spots are due to the various accretion shocks around the star. If these stars are seen at high inclinations, we can expect to observe stochastic occultations of the photosphere by dust lifted above the disk plane near the base of the accretion tongues whenever one passes in front of our line of sight, leading to light curves dominated by aperiodic extinction events. These aperiodic extinction events could also be due to occultation by dust that is lifted above the disk plane as a result of magnetorotational instabilities (Turner et al. 2010).

During a study of the photometric and spectroscopic variability of the CTTS AA Tauri, conducted between 1995 and 2007 (Bouvier et al. 1999, 2003, 2007), a simple geometrical model was presented to explain the behavior of AA Tau's light curve, characterized by a relatively constant level of brightness interrupted by quasi-periodic dips of around 1.4 mag (see Fig. 9 in Bouvier et al. 1999). This variability was attributed to periodic occultations by an inner disk warp spatially associated with two major accretion columns, as in the stable accretion regime. This result provided great support for MHD models.

It is interesting to note that after over 20 years of observations often showing the same quasi-periodic behavior, AA Tau's light curve suddenly changed quite drastically when its average brightness level decreased by over 2 mag in 2011 (Bouvier et al. 2013), at which point it lost its periodicity. This indicates that, though the inner disk warp structure may be stable over the course of many years, it can disappear in a timescale of less than one year.

There are many possible scenarios in which a warp can appear within the circumstellar disk of a T Tauri star besides the one described here (see, e.g., Terquem \& Papaloizou 2000; Flaherty \& Muzerolle 2010). In this paper, we treat the case of CTTS, which are actively subject to ongoing magnetospheric accretion, with inner disk warps located at or near the disk's corotation radius (see discussion in Sect. 3.5). It is reasonable to assume that a warp at this location is due to the interaction between an inclined magnetosphere and the inner disk, and therefore associated with the accretion columns, as was considered for AA Tau in Bouvier et al. (2007). For the stars in this paper, we will consider this to be the main mechanism responsible for the inner disk warp.

\subsection{The occultation model proposed for AA Tau}

Bouvier et al. (1999) considered that the dynamic interaction between the inclined magnetic field of the star and its inner disk region would cause the material in this part of the disk to be lifted, preferentially where the stellar magnetic dipole is closest 
to the disk, resulting in two arch-shaped walls located opposite to each other. This warp, located at or near the co-rotation radius $\left(R_{\mathrm{co}}\right)$ of the accretion disk, eclipses the stellar photosphere when it passes through the observer's line of sight. It has an azimuthal extension of $\phi_{\mathrm{w}}$ and a maximum height of $h_{\max }$ from the disk's mid plane, which occults the stellar photosphere at phase 0.5 , provoking a minimum in the light curve.

The model uses simple geometric principles to reproduce a synthetic light curve where an optically thick clump of given maximum height $h_{\max }$ and azimuthal extension $\phi_{\mathrm{w}}$, present at the co-rotation radius $R_{\mathrm{co}}$, occults the stellar photosphere of a system seen at inclination $i$. The height of the clump is assumed to vary according to

$h(\phi)=h_{\max }\left|\cos \left(\frac{\pi\left(\phi-\phi_{0}\right)}{\phi_{\mathrm{w}}}\right)\right|$

for values of $-\phi_{\mathrm{w}} / 2 \leq \phi \leq \phi_{\mathrm{w}} / 2$, where $\phi_{0}$ is the azimuth of maximum disk height. The clump height is equal to zero for all values of $|\phi|>\phi_{\mathrm{w}} / 2$. In the case of AA Tau, the warp found to best reproduce its light curve had a maximum height of $h_{\max }=$ $0.3 R_{\mathrm{co}}$, and an azimuthal extension of $360^{\circ}$.

\subsection{AA Tau-like light curves in NGC 2264}

In a study of CTTS in the young cluster NGC 2264 using light curves from the CoRoT satellite (Convection, Rotation and planetary Transits, Baglin et al. 2006), various CTTS with light curves similar to that of AA Tau were found (Alencar et al. 2010). NGC 2264 is a young stellar cluster of approximately $3 \mathrm{Myr}$ situated in the Orion spiral arm, around $760 \mathrm{pc}$ from the Sun (for a review of the cluster, see Dahm 2008). Of the stars in the region observed by the CoRoT satellite in 2008, 301 were confirmed as members of the cluster, and of these 83 were classified as CTTS by Alencar et al. (2010).

The 83 CTTS were separated into three groups, according to the morphology of their light curves. The first group consisted of 28 periodic, nearly sinusoidal light curves, whose variabilities were attributed to long-lived cold spots; the second group consisted of 23 light curves with the same characteristics as those of AA Tau, periodic but with varying amplitude and shape of dips; and the third group consisted of 32 non-periodic light curves. Two examples of the AA Tau-like light curves, shown in full and folded in phase, can be seen in Fig. 2 .

In the same study that identified these light curves, Spitzer IRAC single epoch photometry was used to determine whether there is dust in the inner disk region of these stars, by identifying near-infrared excess emission. Of the 83 CTTS, 68 were also observed by Spitzer. All of the stars with AA Tau-like light curves that were observed by Spitzer and CoRoT were shown to present dust in the inner disk region. This is consistent with the assumption that the photometric behavior of these stars is due to obscuration by optically thick material in their inner disks.

In order to better understand the physical processes that govern CTTS variability, a new observational campaign of NGC 2264 was organized in December 2011. The Coordinated Synoptic Investigation of NGC 2264 (CSI 2264, Cody et al. $2014)^{1}$ is composed of simultaneous observations on fifteen telescopes (eleven ground-based and four space-based telescopes), including the CoRoT satellite, the Spitzer Infrared Array Camera

\footnotetext{
1 The CoRoT and Spitzer light curves for all probable NGC 2264 members, as well as our broad band photometry for these stars, are available at http://irsa.ipac.caltech.edu/data/SPITZER/ CSI2264.
}
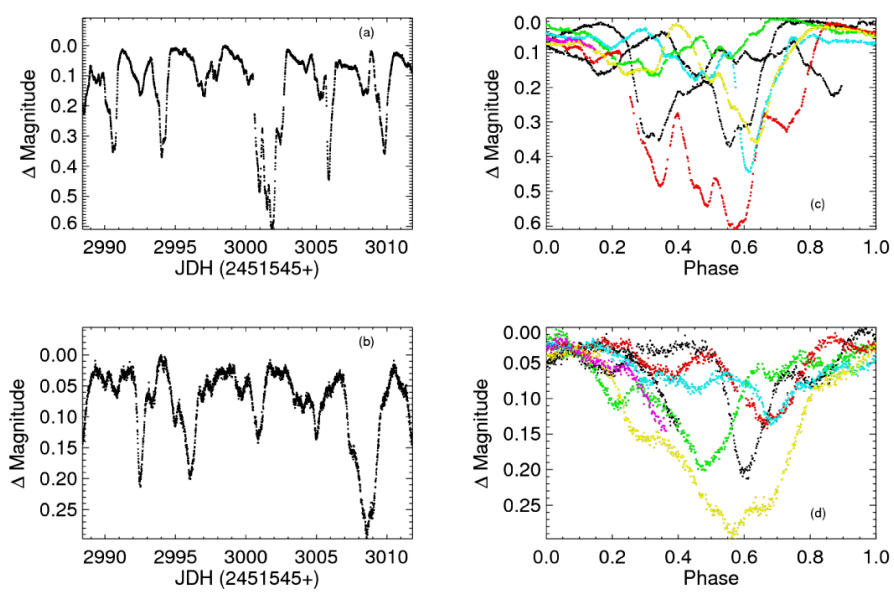

Fig. 2. Two examples of AA Tau-like light curves found for CTTS in NGC 2264 by Alencar et al. (2010). c) and d) show the light curves a) and b), respectively, folded in phase. Different colors correspond to different rotation cycles.

(IRAC, Fazio et al. 2004), the Canada-France-Hawaii Telescope (CFHT) MegaCam (Boulade et al. 2003), and the FLAMES multi-object spectrograph on the Very Large Telescope (VLT, Pasquini et al. 2002). This campaign provided us with simultaneous photometric and spectroscopic information in a wide range of wavelengths. New CTTS were identified according to their UV excess (Venuti et al. 2014), and various new stars with AA Tau-like light curves were found, in addition to stars with newly identified types of photometric behavior (Stauffer et al. 2014, 2015).

Because of the large number of previous studies of NGC 2264, it was possible to establish reliable criteria for cluster membership, as discussed in Alencar et al. (2010). As probable cluster members, we used photometric $\mathrm{H} \alpha$ and variability from Lamm et al. (2004) following their criteria; X-ray detection from Ramírez et al. (2004) and Flaccomio et al. (2006) and location on the cluster sequence in the $(I, R-I)$ diagram, when available; spectroscopic $\mathrm{H} \alpha$ equivalent width greater than a spectral type dependent threshold (White \& Basri 2003); and $\mathrm{H} \alpha$ emission line width at $10 \%$ intensity greater than $270 \mathrm{~km} \mathrm{~s}^{-1}$, as proposed by White \& Basri (2003) to identify accreting T Tauri stars.

In this study we present a multi-wavelength analysis of the CTTS members of NGC 2264 that appear to have extinction dominated photometric behavior. In Sect. 2 we present the observations used in this study, while in Sect. 3 we show the analysis of our data and our results. In Sect. 4 we present a discussion on the proposed cause of these stars' photometric variabilities, and in Sect. 5 we present our conclusions.

\section{Observations}

\subsection{CoRot data}

The first CoRoT short run occurred in March 2008, when the young cluster NGC 2264 was observed for 23 days uninterruptedly. Nearly four years later, during the CSI 2264 campaign, the cluster was observed once again by CoRoT from 2011 December 1, to 2012 January 3, during the satellite's fifth short run. CoRoT's field of view is larger than the full extent of the cluster, which fits entirely into one of the CCDs designed for studying exoplanets. Stars of magnitude down to $R \sim 18$ were observed, with a cadence of 512 s. In some cases the data were 
taken with exposure times of $32 \mathrm{~s}$ to provide high cadence light curves. All of the light curves from the first epoch were rebinned to $512 \mathrm{~s}$.

CoRoT observes in a white light bandpass of approximately $3700 \AA$ to $10000 \AA$. It can, in principle, provide three-color photometry by passing the stellar light through a low-resolution spectral dispersing prism, but for the purpose of this paper we used only the integrated white light flux.

After undergoing standard CoRoT pipeline procedures, detailed in Samadi et al. (2006), the data were subsequently processed to remove unwanted effects, such as those caused by Earth eclipses, and to remove measurements affected by the South Atlantic Anomaly, using a sigma-clipping filter. Care was taken not to remove flaring events. In some cases, jumps due to detector temperature jumps were present in the photometry, and when possible they were removed manually (for more details on the correction of CoRoT light curve systematics, see Cody et al. 2014).

\subsection{Spitzer IRAC data}

The Spitzer Space Telescope mapped a region of $\sim 0.8^{\circ} \times$ $0.8^{\circ}$ centered at RA 06:40:45.0, declination +09:40:40, from 2011 December 3, to 2012 January 1. Targets were observed approximately twelve times a day in the IRAC $3.6 \mu \mathrm{m}$ and $4.5 \mu \mathrm{m}$ channels in its Warm Mission mode. Most of the objects have data from both bands, but around $40 \%$ of the objects fall near the edges of the mapping region, and only have data from one band. A staring mode was also used around the beginning of the run for a region near the center of the cluster. A cadence of approximately $15 \mathrm{~s}$ was obtained during four blocks of 20, 26, 16, and $19 \mathrm{~h}$, on 2011 December 3, December 5, 6, December 7, 8, and December 8, 9 .

The IRAC data reduction procedures and production of IRAC light curves are explained in detail in Cody et al. (2014) and Rebull et al. (2014).

\subsection{CFHT MegaCam data}

NGC 2264 was observed using the Canada France Hawaii Telescope's wide-field imager MegaCam (Boulade et al. 2003) between 2012 February 14 and 2012 February 28. The MegaCam's field of view spans a full $1 \times 1$ square degree, covering the cluster with only one pointing. Data were taken in the $u$ and $r$ bands on 30 epochs. Reduction of these data is explained in Venuti et al. (2014).

\subsection{USNO data}

On the order of 900 epochs of I-band photometric observations of NGC 2264 were obtained at the US Naval Observatory 40" telescope from December 2011 to March 2012. A field of view of $23^{\prime} \times 23^{\prime}$, centered approximately on the cluster center, was observed. The data underwent standard reduction procedures using bias and dome flat images that were obtained at the beginning of each night. Aperture photometry was performed for all stars in each image, and a set of non-variable stars was identified in order to establish zero-points for each CCD frame and construct light curves of the targets using differential photometry.

\subsection{Spectroscopy}

For some stars in our sample, 20-22 epochs of VLT FLAMES spectra were obtained between 2011 December 4, and 2012 February 29. Of these, we have four to six epochs of simultaneous FLAMES spectroscopy and CoRoT photometry.

FLAMES can obtain medium- and high-resolution spectra for multiple objects over a field of view 25 arcmin in diameter. Since this is much smaller than the extent of NGC 2264, even with two pointings, only the central part of the cluster was observed (one pointing centered at RA 06:41:04.8, declination +09:45:00, the other centered at RA 06:40:58.8, declination +09:21:54). We used the HR15N grating of the GIRAFFE/MEDUSA mode, which covers H $\alpha$ and Li $6707 \AA$ with a resolution of 17000 . A few of the brighter stars were observed with the UVES (Ultraviolet and Visual Echelle Spectrograph) red mode, which covers the region from $4800 \AA$ to $6800 \AA$ with a resolution of 38700 . Only one of the stars treated in this paper was observed with UVES.

Where FLAMES spectra were not available, we used highresolution Hectochelle spectra from 2004-2005 provided by Gábor Fûrész (Fûrész et al. 2006). They used the $190 \AA$ wide order centered at $\mathrm{H} \alpha$, with a resolution of $R \sim 34000$. These spectra were used for eight stars.

\section{Data analysis and results}

\subsection{Initial selection of candidates for extinction dominated light curves}

Throughout this paper we are concerned with stars that present extinction events due to circumstellar material eclipsing the stellar photosphere. This requires that the stars in our sample be CTTS, with at least some optically thick dust still present in the disk. Therefore, after selecting the probable members of NGC 2264 following criteria described in Alencar et al. (2010), a subset of stars with CoRoT observations in either epoch was classified as CTTS based on the following criteria: $\mathrm{H} \alpha$ width at $10 \%$ intensity greater than $270 \mathrm{~km} \mathrm{~s}^{-1}, \mathrm{H} \alpha$ equivalent width higher than a spectral type dependent criterion proposed by White \& Basri (2003), or U-V excess greater than a spectral type dependent threshold described in Alencar et al. (2010). A new group of CTTS candidates proposed by Venuti et al. (2014) based on UV excess measured with the CFHT Megacam during the CSI 2264 campaign was also taken into consideration. We also consider the two stars Mon-56 and Mon-14132 as candidate CTTS since they have IR excess and photometric variability characteristic of the CTTS studied in this paper, though we do not have enough spectral information to classify them as CTTS and they present no considerable UV excess.

Of the $\sim 300$ confirmed members observed in 2008, we classified 95 as CTTS or CTTS candidates, 12 more than had been considered by Alencar et al. (2010). Of the $\sim 500$ confirmed members observed in 2011, 148 were classified as CTTS or candidates. Of all of these CTTS and CTTS candidates, 84 were observed in both epochs. Therefore our initial sample of possible CTTS is of 159 cluster members, observed with CoRoT in either 2008, 2011, or both. Sousa et al. (in prep.) performed an in-depth study of all of these CTTS, focusing on the VLT spectroscopy.

The CoRoT light curves of these 159 stars were separated into different groups based on their visual morphologies. This was done based solely on the CoRoT light curves, with no color information. One of these groups consists of AA Tau-like light curves, which present a relatively stable maximum brightness 



Fig. 3. Examples of different light curve morphologies found in CTTS in NGC 2264. a) and b) show periodic light curves attributed to circumstellar extinction, c) shows an accretion burst dominated light curve, d) shows a light curve dominated by aperiodic extinction, e) shows a spot-like light curve, and f) shows a light curve whose morphology is very complex, probably as a result of a combination of processes.

level interrupted by quasi-periodic flux dips that are neither spotlike nor eclipse-like in nature. The term quasi-periodic means that the flux dips present a stable period but vary in shape and/or amplitude between rotation cycles. They vary by on average $25 \%$ in width and $30 \%$ in amplitude from one rotation cycle to the next. Their periods range from 3 to 10 days and are consistent with Keplerian rotation near the co-rotation radius (see Fig. 13 and discussion in Sect. 3.5). They are usually accompanied by other narrower, shallower flux dips that are not necessarily periodic and can have significant structures within the main flux dips.

Bouvier et al. (2007) showed that the photometric behavior of the star AA Tau varied significantly between observations separated by a few years, showing at times the quasi-periodic flux dips attributed to the inner disk warp, at times more than one flux dip per stellar rotation period, and at times with almost no variability (see Fig. 15 in Bouvier et al. 2007). The light curves we have described as AA Tau-like are based on the observations of AA Tau from 1995. There are, however, some light curves that are similar to the 1995 AA Tau light curve, but that either do not have a well-defined maximum brightness, or show relatively weak periodicity (for example, see star Mon-1054 in Fig. 20). Cody et al. (2014) do not classify these as AA Tau-like light curves, but they are important in the context of this paper because they may represent systems that are undergoing events similar to those that AA Tau underwent during its other phases. Therefore, we consider stars that present these light curves in one CoRoT observation or the other to be candidate AA Tau systems along with the stars that have classical AA Tau-like light curves. We analyze their photometric variability in the same way as we do the AA Tau-like light curves, in order to establish whether the stable accretion mechanism viewed at a high inclination could also be responsible for their variability.

Another group that is of interest to this study is composed of light curves with similar characteristics to the AA Tau-like light curves, but that show no obvious periodicity. These light curves appear to be dominated by aperiodic extinction events. These two groups can easily be distinguished from the periodic, nearly sinusoidal, variability attributed to long-lived cold spots, or the nearly symmetric, narrow flux bursts described in Stauffer et al. (2014) and attributed to stochastic accretion events. A few examples of different types of variability are shown in Fig. 3. For a detailed discussion of these and other light curve morphologies, including a statistical analysis of symmetry in the flux and periodicity, see Cody et al. (2014).

Figure 4 shows the CoRoT light curve and USNO I-band light curve, shifted to coincide in magnitude, of two AA Tau-like stars. It is evident from this figure, though the $I$-band photometry coincides closely with the CoRoT photometry, that the lower cadence makes it difficult for AA Tau-like behavior to be identified using only ground-based data. This shows the importance of high cadence space-based photometry to better understand the phenomena that occur among these young stellar objects.

Table 1 shows information on the stars in our sample that are considered to have AA Tau-like or aperiodic extinction dominated light curves in either epoch. The CSIMon ID is an internal naming scheme devised for the CSI 2264 campaign (see Cody et al. 2014), and comprises all cluster members, candidates and field stars in the NGC 2264 field of view (for brevity, throughout the text, we eliminate 'CSI' and the leading zeros and replace, for instance, CSIMon-000250 with Mon-250). The CoRoT ID is the identification number assigned by the CoRoT satellite to all 

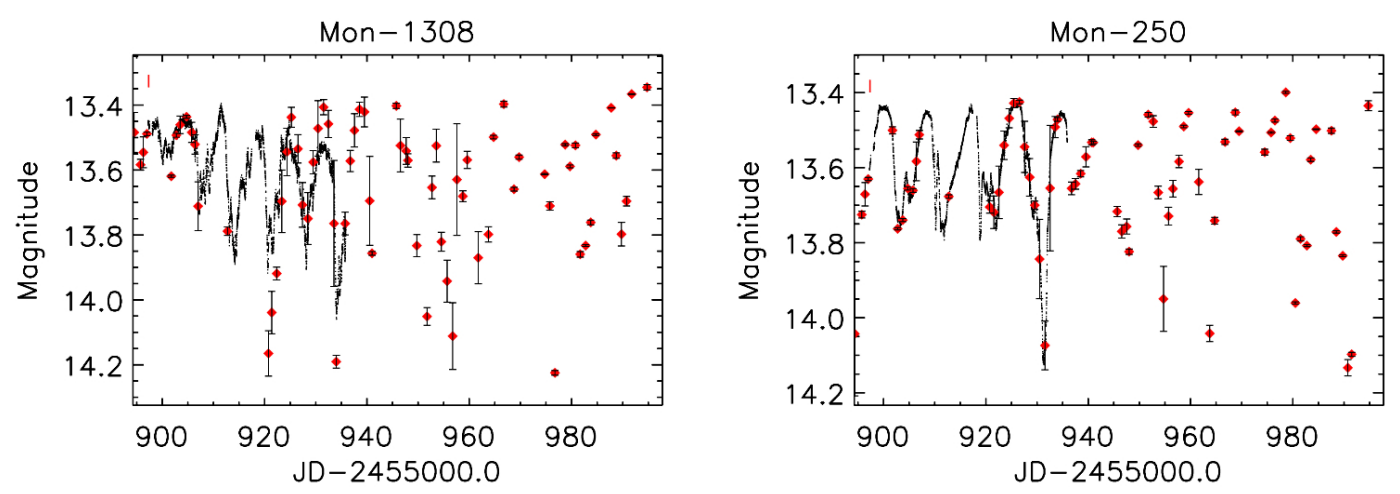

Fig. 4. I-band and CoRoT photometry, shifted in magnitude to coincide, for the AA Tau-like stars Mon-1308 and Mon-250. The high cadence space-based observations allow the AA Tau-like flux dips to be easily identified, while with even high quality ground-based photometry it is difficult to obtain enough information for the variability to be quantified.

objects it has observed. In some cases where the same star was observed in both epochs, two different CoRoT IDs were assigned to the same star. For these stars we present the ID from the 2008 observation. The 2MASS identification is also presented.

The sixth and seventh columns of Table 1 show how the light curve was classified in one epoch or the other. Those labeled with AAT present AA Tau-like light curves, those labeled with AATc are those that were not classified as AA Tau-like by Cody et al. (2014), but that we consider to be candidate AA Tau systems, and those labeled as Ap show aperiodic light curves. For six stars, a possible AA Tau-like variability was found in both epochs, meaning the mechanism responsible for this variability can be stable on a timescale of a few years. Four stars that present AA Tau-like variability were only observed in one epoch. There are thirteen stars that were observed in both epochs and classified as AA Tau-like in one and aperiodic in the other. Of these, five displayed AA Tau-like behavior in 2008, yet in 2011 presented aperiodic variability, while the other eight showed the opposite trend. This points to a possible change from a stable accretion regime to one of unstable accretion (or vice-versa) in a matter of less than four years. This is discussed in Sect. 3.3. Another five stars were classified as having an aperiodic extinction dominated light curve in both epochs. Five more stars with aperiodic extinction dominated light curves were only observed in 2011.

It is interesting to note that, though many stars suffered a transition between AA Tau-like and aperiodic extinction dominated light curves, no star whose light curve was classified as AA Tau-like in one CoRoT observing run was classified as spotlike in the other. This could be due to inclinations, since high inclinations would favor extinction events and possibly hide any variability due to spots on the stellar surface.

Another group of stars with what appear to be short duration extinction events present in their CoRoT light curves is studied by Stauffer et al. (2015). These stars present narrow flux dips that are generally shallower than in AA Tau-like light curves, and nearly Gaussian shaped, while AA Tau-like light curves usually show very irregularly shaped dips, often with significant structure in the minima. The narrow dip stars are periodic over timescales of tens of days, with periods that are consistent with Keplerian rotation periods of the inner disk region. Stauffer et al. (2015) argue that the inner disk warp is not adequate to explain these flux dips because of their short durations and regular shapes, and propose alternate scenarios to account for the variabilities. Two of the stars in our sample of AA Tau-like light curves are considered in their paper as well. They are Mon-56 and Mon-1131, and they present a rather complex photometric behavior. Therefore they are studied therein through different points of view, in order to investigate other possible causes for their variabilities. There are also some stars with AA Tau-like light curves that at times present these narrow flux dips along with the broader ones. These features of their light curves are also discussed in Stauffer et al. (2015).

\subsection{Veiling and variability}

\subsubsection{Correlation between photometric variability and veiling}

Veiling occurs as a result of a strong UV and optical excess produced in the accretion shocks on the stellar surface of CTTS. It is present in spectra when the hot spot caused by these accretion shocks is visible to the observer. This UV excess results in an added blue continuum to the photosphere's spectra, resulting in somewhat shallower absorption lines than those from a pure photosphere. For the CTTS in NGC 2264 for which we obtained FLAMES spectra, we measured the veiling and its variability, using the equivalent widths of the Li I $6707.8 \AA$ and Fe I $6633.4 \AA$ lines. These lines were chosen since they are the absorption lines with the best signal-to-noise ratio in our spectra. As veiling increases, the equivalent widths we measure will decrease.

Many of these CTTS show a certain amount of variability in their measured veiling. We verified whether there was a correlation between this variability and the photometric variability. In principle, a correlation or anti-correlation should exist in some cases, depending on the physical processes occurring on the stellar photosphere. If, for instance, the variability is due to obscuration of the photosphere by an inner disk warp that is associated with accretion columns, we can expect two possible scenarios. When the accretion shocks on the stellar surface are not visible, or the veiling is too small to be accurately measured, there will be no considerable variability in the observed line equivalent widths. If, on the other hand, accretion shocks appear in our line of sight and produce measurable veiling, they should appear along with the inner disk warp, since both are associated with the accretion columns. In this case, when the inner disk warp occults the star causing brightness minima, the veiling should increase thanks to the appearance of the hot spots caused by accretion shocks. Therefore, for an AA Tau-like light curve, the equivalent width of Li I (LiIEW) or Fe I (FeIEW) should either remain relatively constant or increase with increasing brightness. In the latter case, this could be evidence that the star's photometric 
P. T. McGinnis et al.: Probing the inner disks of AA Tau-like systems in NGC 2264

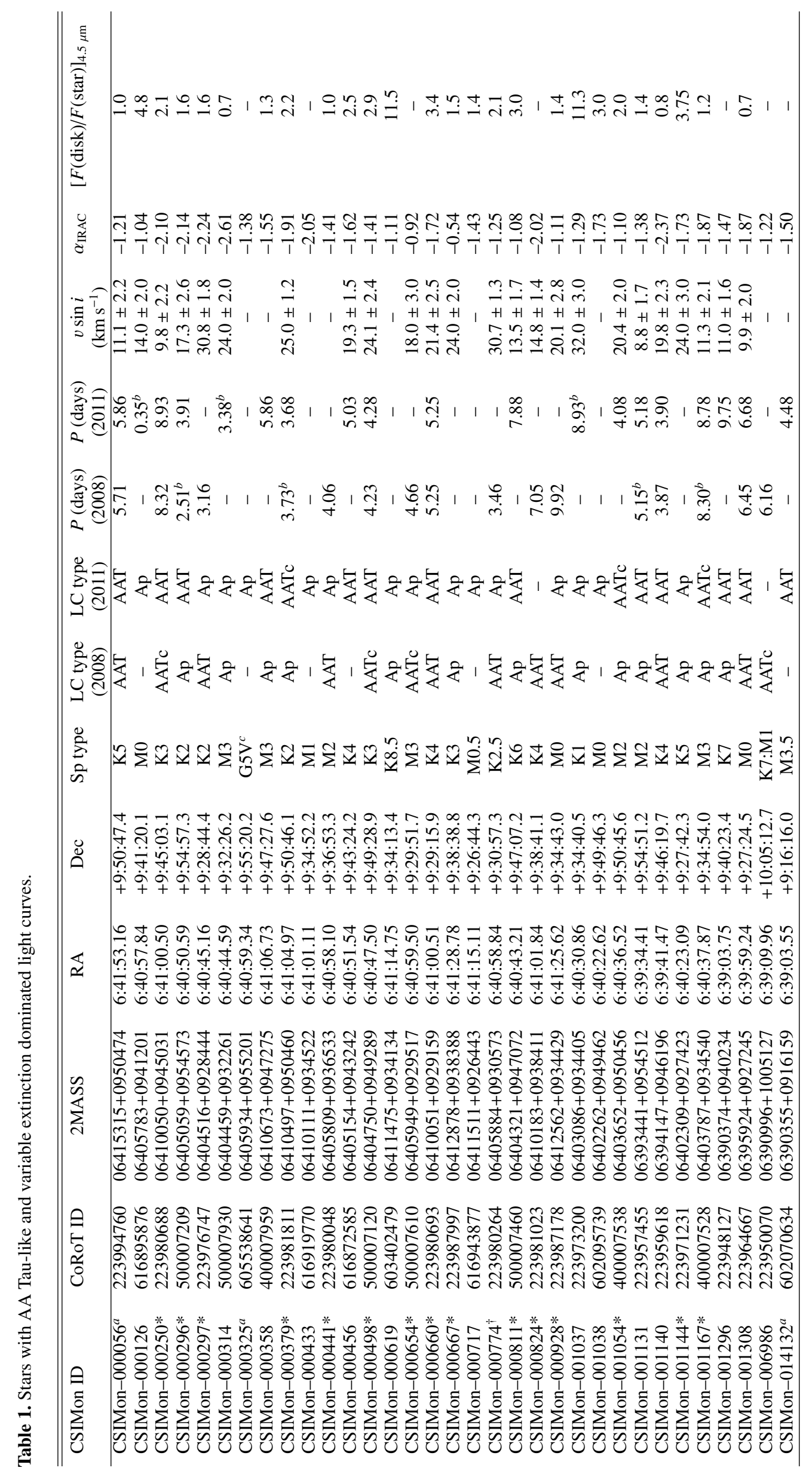

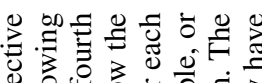



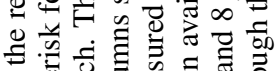


: ष्ठ



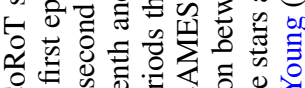

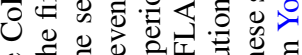

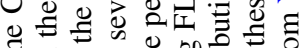




월








बิ

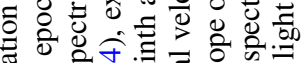

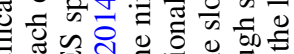

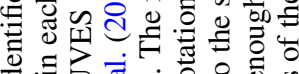





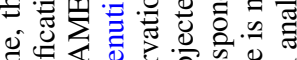

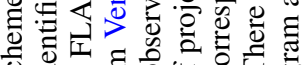

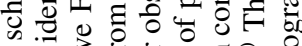
on


पुछ ते च.



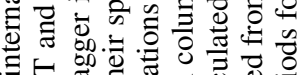

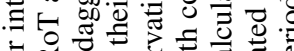
ప융

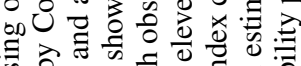

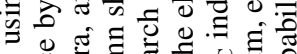


- 0 की 80 过

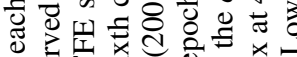


응

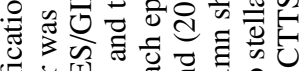

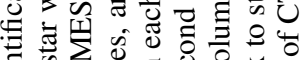





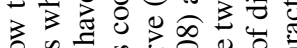

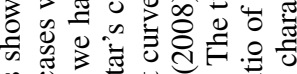

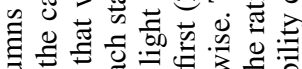

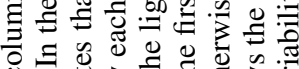
ช

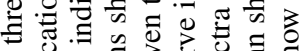

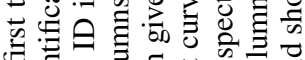

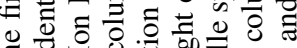

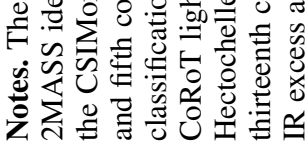



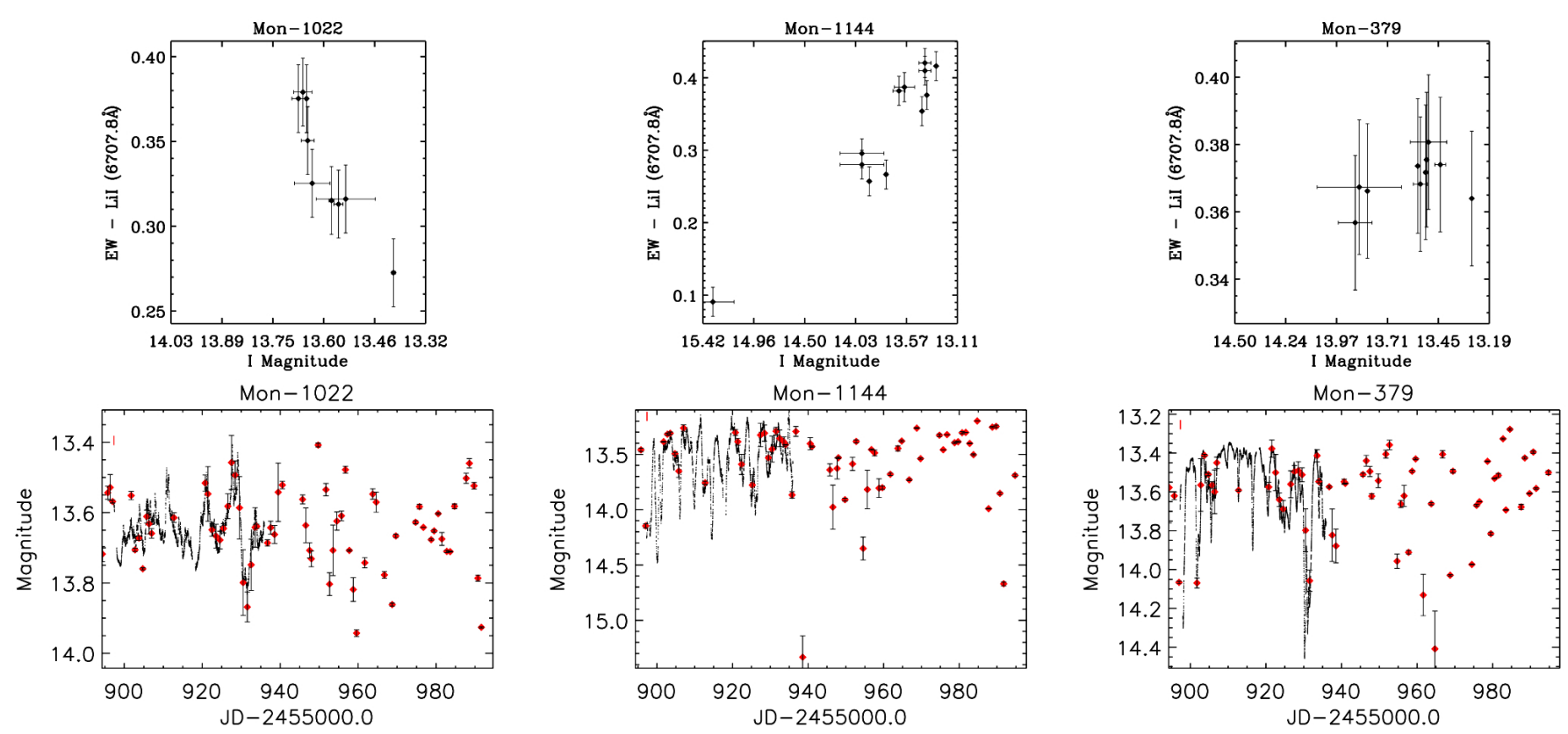

Fig. 5. Top: LilEW vs. I magnitude for three different stars. Bottom: I-band and CoRoT light curves, shifted in magnitude to coincide, of the same three stars. Left panels: the star has an accretion-burst dominated light curve, and veiling variability that supports the variable accretion scenario. Middle panels: the star has a light curve that presents aperiodic extinction events, and veiling variability that supports a scenario in which the occulting structures are associated with accretion shocks. Right panels: the star has a light curve with AA Tau-like characteristics, while its near lack of veiling variability can neither confirm nor refute the hypothesis that it is an AA Tau-like system.

variability is due to obscuration by an inner disk warp that is spatially associated with the accretion columns.

For photometric variability that is due mainly to a configuration of hot spots on the stellar surface, the veiling should increase with increasing brightness, and therefore FeIEW and LiIEW should decrease with increasing brightness. This is the case for the accretion-driven flux bursts discussed by Stauffer et al. (2014). The reoccurring narrow increases in brightness are likely due to an increase in accretion luminosity and are therefore associated with an increase in veiling.

If the star's photometric variability is due simply to the rotational modulation of a stable, cold spot on the photosphere, the veiling would not be expected to vary, since cold spots do not cause veiling.

Measures of veiling using the FeI $6633.4 \AA$ line are much less accurate than LiI $6707.8 \AA$, since it is shallower in these stars and therefore suffers more from noise in the spectra. For this reason veiling was only measured using FeI for a small subset of these stars with FLAMES spectroscopy. In these cases the observed tendencies agree with those determined using the LiI line.

We made plots of LiIEW vs. USNO I-band magnitudes for the stars in our sample where both data were available. The I-band light curves were used rather than the CoRoT light curves because they had better time coverage, despite the lower cadence. There are at most six FLAMES spectra of each star obtained simultaneously with the CoRoT observations, whereas the USNO observations spanned the entire FLAMES campaign. In order to construct the plots, $I$-band measurements taken on the same night were averaged and matched with the equivalent width found using the FLAMES spectrum taken on that night. The USNO and VLT are separated by little more than $40^{\circ}$ in longitude, so the observations were likely taken within a few hours of each other on most nights.
Figure 5 shows plots of LiIEW vs. I-band magnitudes (top panels) for three different examples, along with the corresponding CoRoT and $I$-band light curves (bottom panels). The left panels of Fig. 5 show a star whose veiling increases with increasing brightness. We can see from its light curve that it is dominated by flux bursts attributed to variable accretion events. The middle panels of Fig. 5 show a star whose veiling increases with decreasing brightness, a tendency we attribute to occultation of the photosphere by an inner disk warp associated with the accretion mechanism. Its light curve morphology shows variability that could be explained by this scenario. The right panels of Fig. 5 show a star whose veiling does not vary considerably, which can mean the photometric variability is due to a configuration of cold spots on the surface, or to obscuration by an inner disk warp that either fully occults the accretion shocks associated with it, leaving the veiling unaltered during eclipses, or the accretion is relatively low, and only a small hot spot is present, not capable of generating a large UV excess or veiling. The light curve agrees with the inner disk warp scenario, since cold spots would result in a much more regular photometric variability, but the veiling can neither confirm nor disprove this hypothesis. This star also presents little UV excess, consistent with either of these scenarios.

The star Mon-811 was outside the USNO field, and therefore we have no $I$-band information for it. For this one star, we made a plot of LiIEW vs. CoRoT flux counts instead. This plot was not nearly as well sampled, since very few FLAMES spectra were taken during the CoRoT observations, but it shows a tendency similar to the middle panels of Fig. 5.

Figure 6 shows plots of LiI equivalent width vs. I magnitude for some of the AA Tau-like or aperiodic extinction stars in our sample (plots of the missing stars can be found in Fig. 21). In many cases the veiling variability is very irregular, and we cannot see a clear pattern (e.g., Mon-498). For the star Mon-379 (shown in the right panels of Fig. 5), the veiling is relatively constant. For six stars, we see an apparent decrease in veiling 

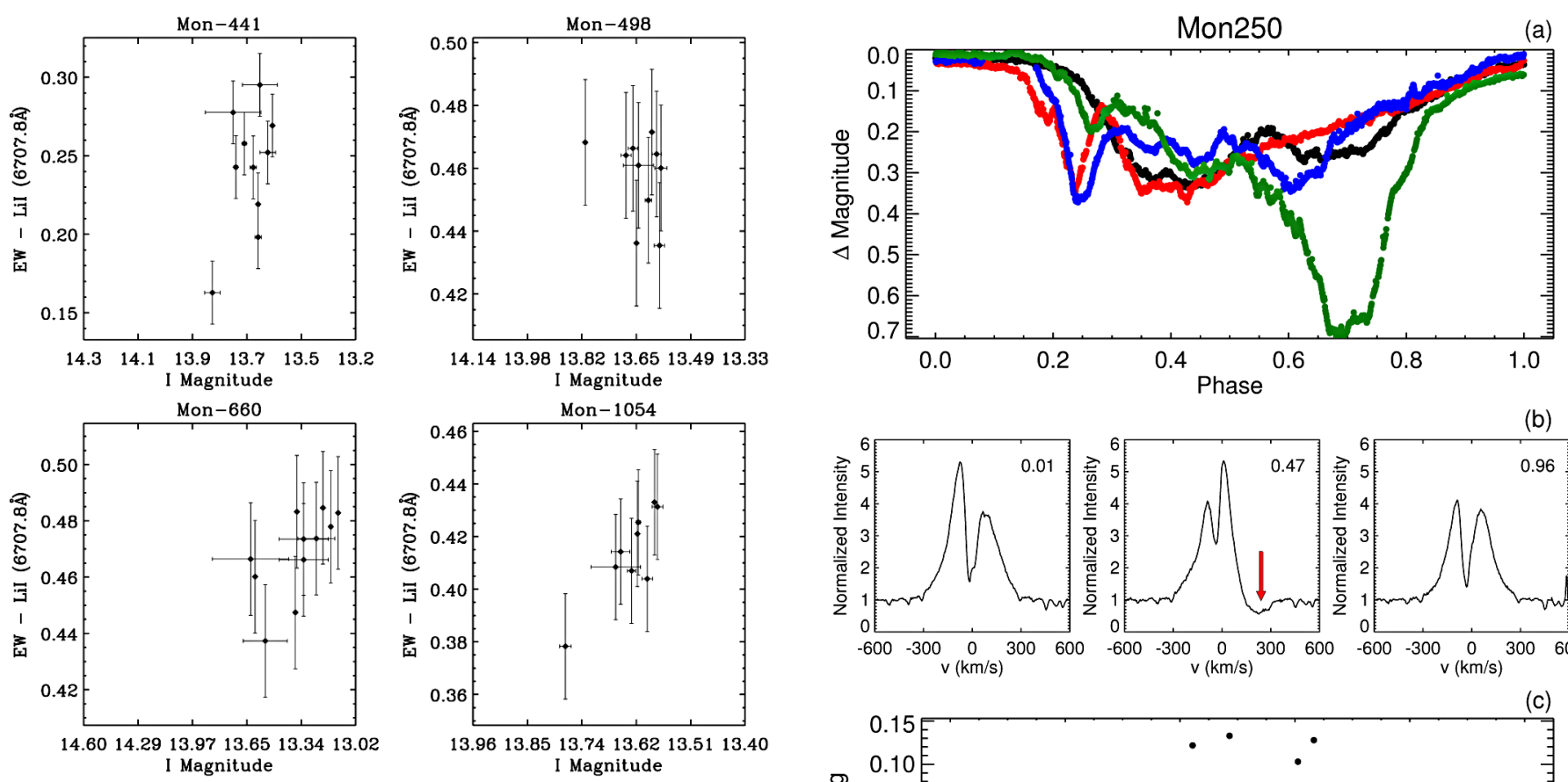

Fig. 6. LiIEW vs. I magnitude for some of the stars in our sample. The star Mon-1054 is an example of a candidate AA Tau system that shows increasing LiIEW with decreasing magnitude. The star Mon-441 shows similar behavior, though its photometric behavior is aperiodic. The star Mon-660 is an AA Tau-like system that appears to show increasing LiIEW with decreasing magnitude, though the uncertainties are quite large, and the star Mon-498 is an example of a system with very irregular veiling that shows no obvious pattern.

(increase in LiI equivalent width) as the star's brightness increases, which could indicate that the mechanism responsible for their photometric variability is obscuration by an inner disk structure associated with accretion columns. This is the case for Mon-250, Mon-441, Mon-660, Mon-811, Mon-1054, and Mon1144. The last has the largest variability amplitude in veiling $(\sim 0.3 \AA)$, meaning there should be significant hot spots on the stellar surface. It is the star with the highest UV excess among the stars in our sample where veiling was measured, which could be expected, since the hot spots associated with the accretion shocks on the surface of the star should produce a considerable UV excess when visible.

Two of the stars that show an increase in veiling during the minima in their light curves have aperiodic photometric variability (Mon-441 and Mon-1144). This provides support for our hypothesis that the occultations in these light curves are caused by dust lifted above the disk plane at the base of unstable accretion streams, since these should be associated with accretion shocks on the stellar surface in a manner analogous to the stable accretion funnels. If these stars are undergoing unstable accretion, they should show no obvious periodicity in their light curves, but if they are seen at high enough inclinations, they should share other similarities with AA Tau-like systems, such as the one we see here.

\subsubsection{The specific case of Mon-250}

We analyzed the $\mathrm{H} \alpha$ line profile of the star Mon-250 during different rotational phases (Fig. 7b), and note a redshifted absorption component (indicated by an arrow in the figure) that appears in several spectra during the light curve minima that is not present in other phases. This is clear evidence that the accretion
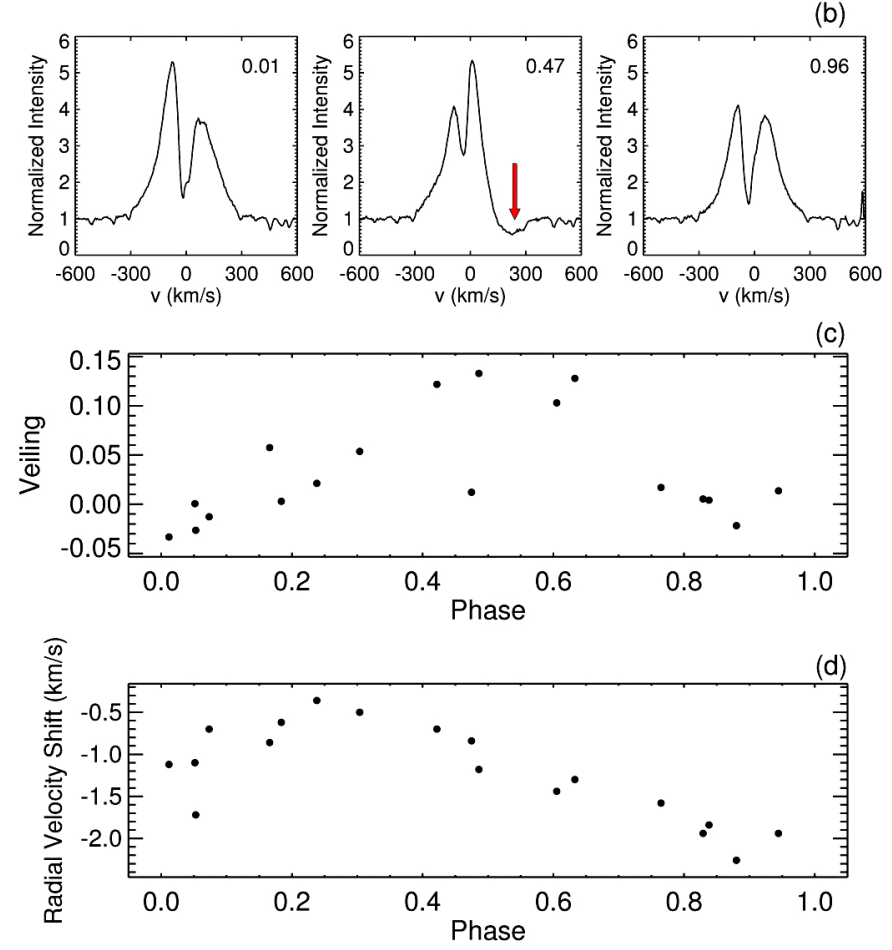

Fig. 7. Mon-250. a) 2011 CoRoT light curve folded in phase. b) $\mathrm{H} \alpha$ line profile during different rotational phases, indicated in each panel. A redshifted absorption component seen only near phase 0.5 is indicated by a red arrow. c) Measured veiling folded in phase. d) Shift in radial velocity measured from photospheric lines in the spectra, folded in phase. In all four panels, the same period of 8.6 days, determined from the radial velocity variation of photospheric lines, was used to calculate the phase. The same initial date was also used.

funnel and hot spot appear in front of our line of sight during the photometric flux dips, since the infalling material responsible for the absorption is moving away from us.

This star's veiling variability also points to the appearance of accretion shocks during occultations. For this particular star, we were able to measure veiling in all 20 FLAMES epochs by comparing line depths to those of a weak line $\mathrm{T}$ Tauri star of the same spectral type, to determine what fraction of the observed continuum should be due to the hot spot. Figure $7 \mathrm{c}$ shows a plot of veiling folded in phase, using the rotation period determined from radial velocity shifts of photospheric lines in the spectra (Fig. 7d). It can be seen that there is almost always an increase in veiling as the light curve undergoes a minimum in brightness. This is evidence of the appearance of a hot spot on the stellar surface, one large enough to produce measurable veiling, during occultations. The rotation period found from the variability of radial velocities measured in the Mon-250 photospheric lines was of $8.6 \pm 0.5$ days, consistent with the period 
found from the CoRoT light curve observed at the same epoch of $8.93 \pm 0.50$ days. This shows that the occulting structure is located at or near the disk's co-rotation radius, as was the case for AA Tau. Unfortunately, this type of analysis was only possible for this star since it was the only one with a high enough variability amplitude in veiling and radial velocity of photospheric lines to draw significant conclusions.

We attribute these phenomena, which were also observed for AA Tau (Bouvier et al. 2007), to the appearance of a major accretion funnel and accretion shock associated with it, at the moment of occultation by the inner disk warp. This shows the association between the inner disk warp and the stable accretion columns, and provides support to our hypothesis that in these cases, the same interaction between the inclined stellar magnetic field and the inner disk that is causing the material to accrete onto the star via two major accretion columns is also responsible for lifting dust from the disk plane, creating a warp in the disk near the co-rotation radius.

\subsection{Stable and unstable accretion regimes}

In this section, we discuss the main differences between the quasi-periodic AA Tau-like stars and the stars whose light curves are dominated by circumstellar extinction, but have no obvious periodicity. We propose that the former may be undergoing accretion via a stable regime, while the latter could be accreting via an unstable regime. Kurosawa \& Romanova (2013) cite the parameters that are important in determining through which of these regimes a star will accrete. They are mass accretion rate $\left(\dot{M}_{\text {acc }}\right)$, truncation radius, stellar rotation rate, the inclination angle $\beta$ between the stellar rotation axis and the axis of the magnetic field ( $\Omega$ and $\mu$, respectively, in Fig. 1 ), and the $\alpha$-parameter, which describes viscosity in the disk.

Kurosawa \& Romanova (2013) mention a possible transition between regimes, which can occur if one or a few of the factors that determine instability change with time within a certain system. For instance, if the truncation radius $R_{\text {mag }}$ becomes smaller than the co-rotation radius $R_{\mathrm{co}}$, due for example to a change in the stellar magnetic field, accretion flows tend to become unstable. The mass accretion rate can also affect the ratio $R_{\mathrm{mag}} / R_{\mathrm{co}}$, since a higher mass accretion rate leads to a smaller truncation radius, therefore leading to a more unstable configuration. The viscosity parameter $(\alpha)$ controls the global mass accretion rate. The higher $\alpha$ is, the higher the mass accretion rate is, leading to more instabilities. According to Kulkarni \& Romanova (2009), the transition from a stable to an unstable regime occurs around critical values of mass accretion rates. They state typical critical values for stars with $R_{\mathrm{mag}} \approx 2-3 R_{*}$ to be around $\dot{M}_{\text {acc }}=1.7 \times 10^{-7}$, and for stars with $R_{\mathrm{mag}} \approx 4-5 R_{*}$, around $\dot{M}_{\text {acc }}=2.1 \times 10^{-8}$.

A smaller angle $\beta$ also leads to less stable configurations, since at large $\beta$ the magnetic poles become closer to the inner disk rim, lowering the potential barrier in the vertical direction that the material must overcome to be lifted into the accretion funnels. According to Kulkarni \& Romanova (2009), values of $\beta>25^{\circ}$ usually lead to stable configurations (see also Romanova et al. 2014).

Venuti et al. (2014) showed that mass accretion rates in CTTS can vary considerably over time. They calculated mass accretion rates for stars in NGC 2264 using data from 2010 December and 2012 January, the latter being nearly simultaneous with the CoRoT observations. They show that many of the stars in our sample had mass accretion rates vary by up to $50 \%$ in
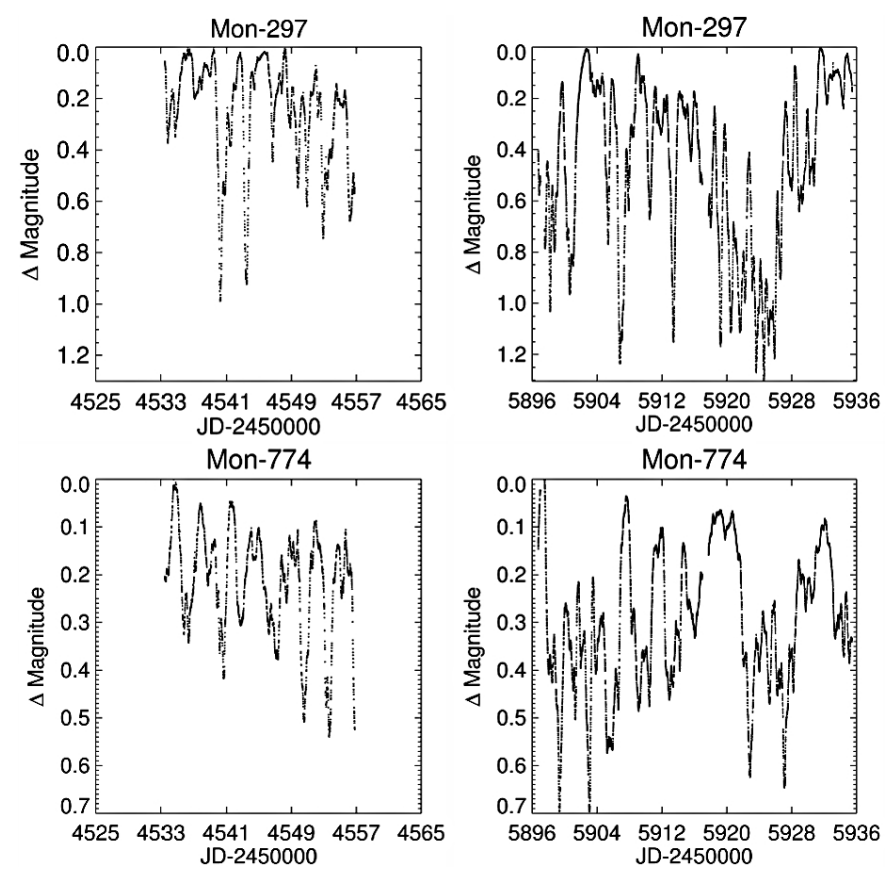

Fig. 8. CoRoT light curves of Mon-297 and Mon-774 during their AA Tau phases (left) and aperiodic phases (right).

little over one year. Unfortunately, there is no estimate of mass accretion rate simultaneous with the 2008 CoRoT observations, but it is possible that a variation such as this contributed to a transition between accretion regimes. However, Venuti et al. (2014) also show that long term changes in mass accretion rates are not necessarily larger than short term changes.

Magnetic field configurations may also be responsible for these transitions. It is possible for the magnetic field of a CTTS to change significantly in a few years. This has been observed, for example, in the CTTS V2129 Oph (Donati et al. 2011). Its magnetic field was measured in two different epochs, nearly four years apart, and the magnetic field strength varied greatly from one epoch to the other. The dipolar component was found to be about 3 times stronger in the second epoch, and the octupolar component 1.5 times stronger than four years earlier. Donati et al. (2011) found that the ratio $R_{\mathrm{mag}} / R_{\mathrm{co}}$ nearly doubled in that time.

In this study we have seen that $38 \%_{-11 \%}^{+14 \%}(5 / 13)$ of the stars that were observed in 2008 as having photometric variability similar to AA Tau were observed again in 2011, showing aperiodic variability due to extinction from circumstellar material. Of the stars that were observed in 2008 as having aperiodic photometric variability due to circumstellar extinction, $62 \%{ }_{-15 \%}^{+11 \%}$ $(8 / 13)$ were observed again in 2011 , then showing variability similar to AA Tau. If our proposed scenario is true, then this points to a transition from a stable to an unstable accretion regime, or vice-versa, in a matter of less than four years, for $57 \%_{-11 \%}^{+9 \%}$ of our sample of possible AA Tau-like stars, i.e., $(5+8) / 23$. An example of two of the stars that appear to have undergone this transition is shown in Fig. 8.

We also performed a periodogram analysis of the light curves of these 13 stars that changed between periodic and aperiodic photometric behavior. The periodograms of each epoch were analyzed separately and compared. For 11 stars, during the epoch of unstable accretion at least a weak signal was present at the period found for the AA Tau phase, believed to be the stellar rotation period. For eight of these, this peak was the only one 



Fig. 9. CoRoT light curves of Mon-928 in 2008 (left) and 2011 (right).

present, or the strongest, despite being much weaker than the one present during the AA Tau phase. One exception is the star Mon-441, which shows a weak signal during its unstable phase, but this signal does not correspond to the period found in the AA Tau phase, or to a multiple of it.

In most cases the variable depths of the flux dip amplitude in a star's light curve during its aperiodic phase are smaller than those measured in its AA Tau phase by $20 \%$ to $60 \%$. However, for 4 of the 13 stars that underwent a transition between AA Tau-like and aperiodic light curve, the flux dip depths remained nearly the same in both epochs, and for 2 more stars the variability amplitude during their aperiodic phase was larger by $20 \%$ to $30 \%$. This means that, at least in some cases, during the unstable accretion phase dust must be lifted above the disk plane at similar, and sometimes even higher, altitudes as during the stable accretion phase.

As for the star Mon-928, the flux dips present in 2008 disappear entirely in 2011. The light curve we observe in the second epoch is not characteristic of circumstellar extinction. The period of 9.92 days found during the first epoch is very similar to periods previously measured for this star in the literature (Lamm et al. 2005), yet it is non-existent in the 2011 light curves (see Fig. 9). One possibility is that in 2011 the inner disk warp did not reach high enough altitudes above the disk to occult the stellar photosphere. At the inclination of $71^{\circ}$ found by us for this system (see Sect. 3.5), our simulations using the occultation model show that if the warp found to best reproduce the 2008 CoRoT light curve decreases in height by at least $26 \%$, the occultations observed in 2008 would no longer occur. This amount of variation in warp height is well within the range we observe for these objects (see Sect. 3.5). This star is also one of the two that appear to have undergone a small increase in mass accretion rate from 2010 December to 2012 January (Venuti et al. 2014); therefore, it is possible that a higher mass accretion rate led to a more unstable configuration during the 2011 observations, resulting in a less pronounced disk warp.

Kurosawa \& Romanova (2013) also predict that for a more unstable regime, one should observe a higher frequency of flux peaks per stellar rotation period in the light curves of stars of medium or low inclinations thanks to the presence of a larger number of accretion shocks on the photosphere. It is fair to assume that, for systems seen at high inclinations, a higher frequency of flux dips would also be observed for more unstable scenarios because of the larger number of accretion streams present around the star, whose base could contain dust that can occult the photosphere. Of the stars in our sample that changed from one regime to the other, most show two to three flux dips per stellar rotation period during the unstable accretion phase, while some show up to seven flux dips per rotation period. This may be an indication of the degree of instability of each star-disk system. The two stars that show the highest frequencies of flux dips during their unstable accretion phases are the two that have the lowest masses in our sample of possible AA Tau-like stars. As is discussed in Sect. 3.7, low mass stars appear to present more unstable accretion, possibly as a result of magnetic field configurations. These higher frequencies of flux dips can also be caused by dust lifted above the disk plane because of magnetorotational instabilities that do not necessarily lead to accretion streams (Turner et al. 2010; Hirose \& Turner 2011).

Of the 23 stars that present AA Tau-like behavior, only six maintain this behavior in both 2008 and 2011. Many appear to show some signs of weak instability even during their AA Tau phase, sometimes presenting two or three flux dips in one rotation cycle. A few examples are Mon-297, Mon-456, and Mon-824 (Fig. 10). Most show many small secondary flux dips present in the light curve, indicating a possible coexistence of secondary accretion streams with the main accretion funnels.

\subsection{Comparing optical and infrared light curves}

Of the 33 stars in our sample of AA Tau-like or aperiodic extinction dominated CoRoT light curves, 29 were observed almost simultaneously with Spitzer in mapping mode. Figure 11 shows a few examples of Spitzer IRAC $3.6 \mu \mathrm{m}$ and $4.5 \mu \mathrm{m}$ light curves, plotted along with the respective CoRoT light curves. The CoRoT flux was transformed into magnitude using its greatest value as magnitude zero, then shifted to overlap the Spitzer IRAC data. Figure 22 shows the light curves of all other stars in our sample.

We see that 4 of the 29 stars show very similar behavior in both bands, and present AA Tau-like modulation in the infrared as well as the optical. These stars are Mon-660, Mon-811, Mon-1140, and Mon-1308. Another five show similar behavior, with minima and maxima generally coinciding in the infrared and optical, but these light curves do not overlap perfectly. These are Mon-297, Mon-314, Mon-433, Mon-441, and Mon-456.

There are nine stars that correlate in some parts of their infrared and optical light curves, while other parts behave differently, or even anti-correlate at times. Stars Mon-56, Mon-126, Mon-358, Mon-654, Mon-717, Mon-774, Mon-1054, Mon-1144, and Mon-1167 show this behavior. The 10 stars Mon-296, Mon-325, Mon-379, Mon-498, Mon-619, Mon-667, Mon-928, Mon-1037, Mon-1038, and Mon-1131 have entirely different infrared and optical light curves, with very little or no features in common.

The star Mon-250 has Spitzer IRAC light curves that almost anti-correlate with its CoRoT light curve. Most of the Spitzer IRAC maxima coincide with CoRoT minima, meaning this variability cannot possibly be the consequence of a hot or cold spot on the stellar surface, since any variability on the surface should be present in all wavelengths. To explain why there is more IR emission during the optical eclipses, we propose the following scenario. The stable accretion regime assumes that there are two main accretion funnels opposite each other, one in each hemisphere. Each of these funnels is associated with a hot spot on the stellar surface, where the accretion shock is located. The base of each accretion funnel is associated with part of the inner disk warp. When one part of the warp occults the stellar photosphere in our line of sight, the hot spot opposite it, as well as the rest of the stellar photosphere on that side of the star, illuminates the corresponding part of the inner disk warp. The dust located there absorbs the light from the hot spot and photosphere and reemits it at longer wavelengths, causing an increase in emission 

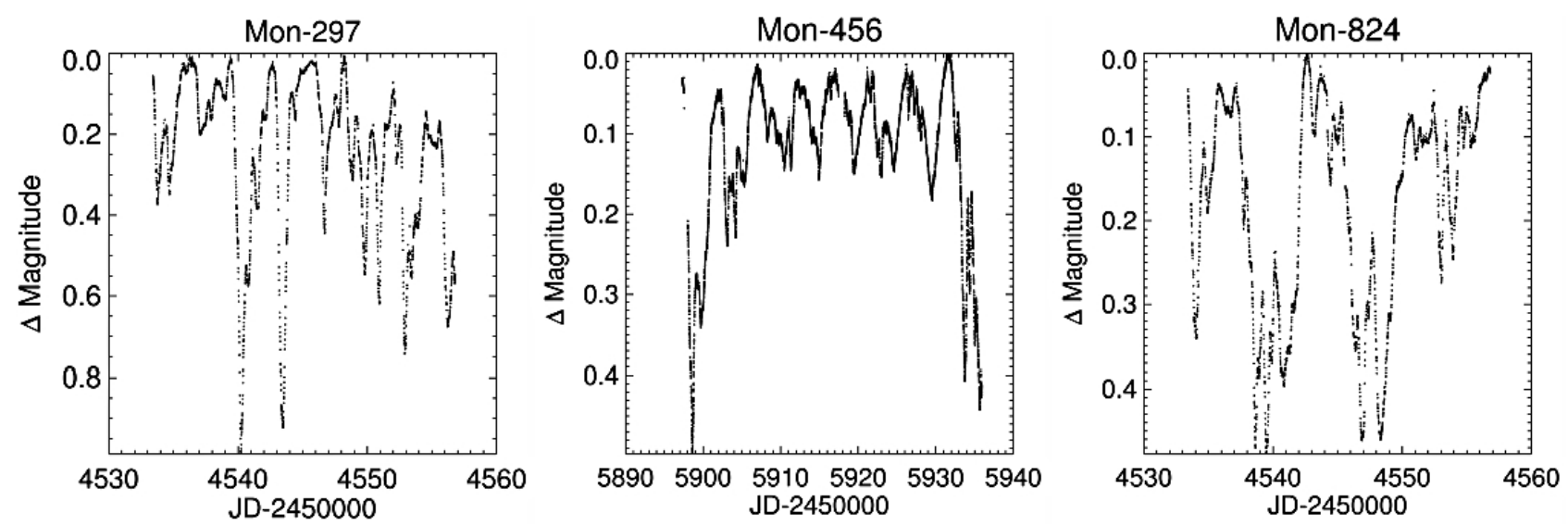

Fig. 10. CoRoT light curves of Mon-297, Mon-456, and Mon-824 during their AA Tau phase. These stars sometimes present two, or even three, flux dips per rotation period, and often secondary flux dips are present alongside the main flux dips, indicating a possible coexistence between unstable accretion streams and stable accretion funnels.
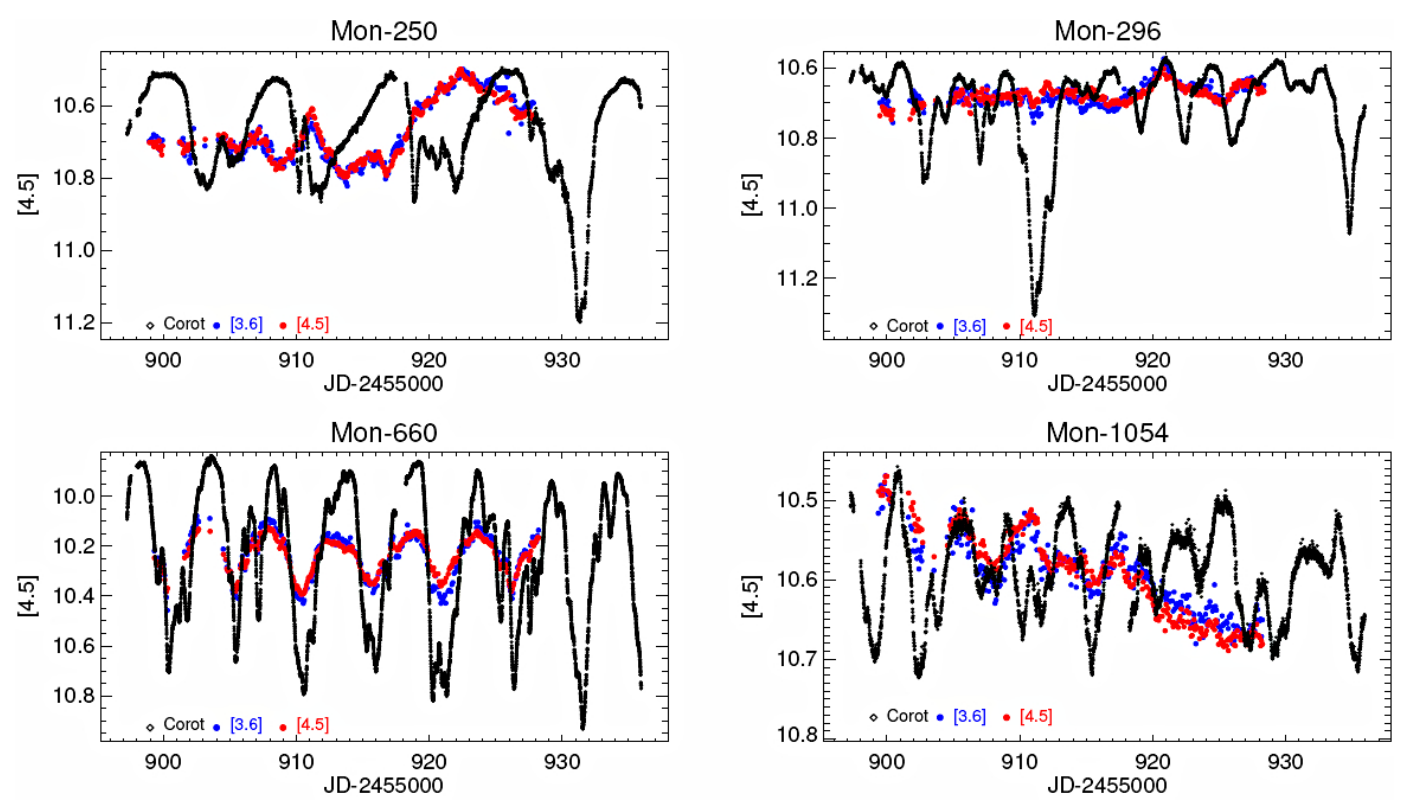

Fig. 11. Spitzer IRAC $3.6 \mu \mathrm{m}$ and $4.5 \mu \mathrm{m}$ light curves (blue and red filled circles, respectively) overplotted on CoRoT light curves (black circles). The CoRoT and IRAC $3.6 \mu \mathrm{m}$ light curves were shifted in magnitude for easier comparison.

Observer
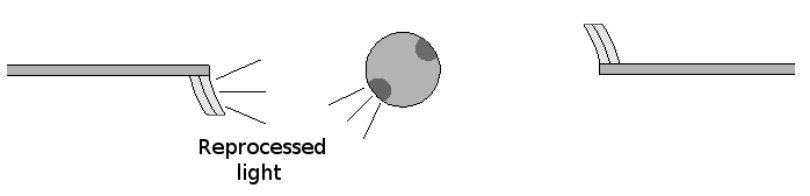

Fig. 12. When one side of the inner disk warp occults the stellar photosphere, the hot spot and photosphere on the opposite side illuminate the part of the warp on that side. The light is absorbed by the dust and is re-emitted at longer wavelengths.

in the infrared (Fig. 12). This emission in the infrared is predicted by the radiation transfer model of Whitney et al. (2013) when applied to an accretion hot spot associated with an inner disk warp. This behavior is also observed in a few other light curves, during one or two rotation cycles (see, e.g., Mon-1054 in Fig. 11, between MJD 55909 and MJD 55 913).
The $\alpha_{\text {IRAC }}$ index is a good indicator of the amount of dust present in the inner accretion disk. It corresponds to the slope of the spectral energy distribution (SED) between $3.6 \mu \mathrm{m}$ and $8 \mu \mathrm{m}$. Unfortunately, there were no Spitzer IRAC observations in $8 \mu \mathrm{m}$ simultaneously with the 2011 CoRoT-Spitzer campaign, since by this time Spitzer was already operating in its warm mission. We do, however, have $\alpha_{\text {IRAC }}$ values from Teixeira et al. (2012) for 14 of these 29 stars with simultaneous CoRoT and Spitzer light curves, and archival IRAC and Multiband Imaging Photometry for Spitzer (MIPS) data (for details see Cody et al. 2014), which enabled us to calculate values of $\alpha_{\text {IRAC }}$ for the stars that were not included in Teixeira et al. (2012). These values are given in Table 1. Lada et al. (2006) established a criterion to classify the inner disk structures of systems according to the $\alpha_{\text {IRAC }}$ index. Stars with $\alpha_{\text {IRAC }}<-2.56$ are classified as naked photospheres (systems devoid of dust within a few $0.1 \mathrm{AU}$ ), those with $-2.56<\alpha_{\text {IRAC }}<-1.80$ have anemic disks (their inner disk region is optically thin), those with $-1.8<\alpha_{\text {IRAC }}<-0.5$ have optically thick inner disks, stars with $-0.5<\alpha_{\text {IRAC }}<0.5$ are flat 
spectra sources, and stars with $\alpha_{\text {IRAC }}>0.5$ are Class I protostellar objects.

We note that the objects in our sample whose CoRoT and Spitzer light curves correlate best have values of $\alpha_{\text {IRAC }}$ between -1.4 and -1.9 , meaning they are generally in the transition region between optically thin and optically thick inner disks. If there is too much dust within a few AU of the star (higher values of $\alpha_{\text {IRAC }}$ ), the Spitzer light curves would be dominated by emission from warm circumstellar dust, rather than eclipses of the stellar photosphere. On the other hand, if the portion of the disk occulting the star is not optically thick, then the flux dips at IRAC wavelengths could be much shallower than for the case where we assume optically thick absorption at all bands. This seems to be the case for Mon-296, whose Spitzer light curve remains relatively steady except for a very shallow dip occurring at the same time as the optical light curve's lowest minimum, and a small burst near one of its maxima (top right panel in Fig. 11). This star's $\alpha_{\text {IRAC }}$ shows that it has an anemic disk. The star Mon-250, mentioned in the discussion above, also has an anemic disk. It has a value of $\alpha_{\mathrm{IRAC}}=-2.10$, one of the lowest in our sample.

Another useful quantity is the ratio of the disk flux to the stellar flux at $4.5 \mu \mathrm{m}$, estimated from the slope of each star's SED as compared to an estimate of the photospheric flux based on the star's spectral type and the Kurucz-Lejeune models (Lejeune et al. 1997), normalized to the observed $J$-band and assuming an appropriate amount of reddening customized to each object. This indicates what fraction of the $4.5 \mu \mathrm{m}$ flux originates at the disk, and should relate directly to how well the light curves correlate. If this ratio is zero, then the optical and infrared light curves should coincide. If it is considerably larger, very little of the infrared flux comes from the star, and the flux dips present in the optical light curves should be absent from the infrared light curves. The values of $F_{4.5 \mu \mathrm{m}}$ (disk) $/ F_{4.5} \mu \mathrm{m}$ (star) estimated for our stars are shown in Table 1.

We see some of these predicted tendencies, but the relationship is not as direct as one would expect. The stars with the lowest fractions of disk flux at $4.5 \mu \mathrm{m}$ tend to have well correlated light curves, and generally very similar variability amplitudes in the optical and infrared, while those with a very high value of disk flux per stellar flux have uncorrelated, or weakly correlated, optical and infrared light curves. For the intermediate values, however, we find all sorts of scenarios. Mon-660, for instance, has an estimated ratio of disk flux to stellar flux of $F_{4.5 \mu \mathrm{m}}($ disk $) / F_{4.5 \mu \mathrm{m}}($ star $)=3.4$, and very strongly correlated light curves, while for Mon-928 $F_{4.5 \mu \mathrm{m}}$ (disk) $/ F_{4.5 \mu \mathrm{m}}($ star $)=$ 1.4 , and its optical and infrared light curves are completely different. This could be due to uncertainties in the SED fits caused by poor or time variable data, or could simply be due to the time variable nature of the SEDs. There could have been some changes in the disk emission between the Spitzer cryogenic mission, from whence the values used to plot the SEDs were taken, and the 2011 CoRoT and Spitzer observing campaign.

For the stars where a correlation can be identified, we calculated which extinction law is necessary to transform the optical to the infrared light curve, assuming an optically thin regime. Extinction laws $A_{\lambda} / A_{R}$, where $\lambda=3.6 \mu \mathrm{m}$ and $4.5 \mu \mathrm{m}$, were found to be between 0.28 and 0.77 . According to Cardelli et al. (1989), we can derive values of $A_{\lambda} / A_{V}$ for a given $R_{V}$ (where $R_{V}$ is defined as $A_{V} / E(B-V)$ ). A value of $R_{V}=3.1$, typically used for interstellar matter (ISM), translates to $A_{3.6} / A_{V}=0.05$ and $A_{4.5} / A_{V}=0.04$. Transforming to $A_{R}$, since the CoRoT filter is centered close to the center of the $\mathrm{R}$ Johnson filter, we would have $A_{3.6} / A_{R}=0.07$ and $A_{4.5} / A_{R}=0.05$. These extinction laws are much lower than those found for the stars in our sample, implying that the distribution of dust grains in the inner circumstellar disk region is quite different from ISM, possibly containing fewer small grains. In addition, the observed dip depth ratio should be corrected for the fraction of IR light emitted by the disk. This would only increase our values of $A_{\lambda} / A_{R}$.

\subsection{Modeling the CoRoT light curves}

In this section we use the occultation model proposed for AA Tau in Bouvier et al. (1999) (described in Sect. 1.1) to attempt to reproduce the AA Tau-like light curves in our sample. If the optical photometric variability observed is due to periodic occultations by an inner disk warp, as we propose, then this geometrical model should be capable of reproducing the general trend of these light curves. We vary the model's main parameters in an attempt to obtain the configuration that most closely matches the observed variability. In this way we determine which values of warp height and azimuthal extension are needed to explain these stars' variability and how they must change from one rotation cycle to another in order to account for the changes in width and amplitude of each flux dip (see also Fonseca et al. 2014, for a detailed study of the light curve of V354 Mon-Mon-660).

As was mentioned in Sect. 1.1, the parameters of the occultation model are the star-disk system's inclination $i$, the radius $R_{\mathrm{w}}$ at which the warp is located, and the warp's maximum height $h_{\max }$ and azimuthal extension $\phi_{\mathrm{w}}$.

We assume that the periods we measured from the CoRoT light curves are the Keplerian periods of the occulting material orbiting the star; therefore, if we know the star's mass, we can use Kepler's third law in the following form to calculate the radius at which the warp is located,

$R_{\mathrm{w}}^{3}=G M_{*}\left(\frac{P_{\mathrm{kep}}}{2 \pi}\right)^{2}$,

where $M_{*}$ is the star's mass, $P_{\text {kep }}$ is the Keplerian rotation period, and $G$ is the gravitational constant. To calculate the inclination we use the following relation between $v \sin i$, stellar radius $R_{*}$, and stellar rotation period $P_{\text {rot }}$ :

$v \sin i=\frac{2 \pi R_{*}}{P_{\text {rot }}} \sin i$.

We used stellar masses and radii determined by Venuti et al. (2014), who compared effective temperatures and bolometric luminosities placed on the Hertzsprung-Russell diagram to premain sequence model grids of Siess et al. (2000). The values are shown in Table 2, along with our values of Keplerian periods found from CoRoT light curves in each epoch, and the corresponding Keplerian rotation radius.

Values of the stars' projected rotational velocities $(v \sin i)$ were obtained comparing FLAMES spectra, when available, and Hectochelle spectra otherwise, to synthetic spectra using the program Spectroscopy Made Easy (SME, Valenti \& Piskunov 1996) and Oleg Kochukhov's Binmag $3^{2}$, as well as the spectrum synthesis code Synth3 (Kochukhov 2007) and atomic line files extracted from the Vienna Atomic Line Database (VALD) ${ }^{3}$ (Piskunov et al. 1995; Ryabchikova et al. 1997; Kupka et al. 1999, 2000). We used photospheric lines to estimate the values of $v \sin i$ shown in Tables 1 and 2. For a few of our stars either no

\footnotetext{
2 http://www.astro.uu. se/ oleg/

3 http://vald.astro.uu.se/
} 




Fig. 13. Period histogram of CTTS in NGC 2264 whose CoRoT light curves were classified as AA Tau-like (blue) or spot-like (red). The periods of spot-like light curves represent stellar rotational periods, while those of AA Tau-like light curves represent Keplerian rotation periods at the warp location. The periods of spot-like light curves were taken from the 2011 data, while AA Tau-like light curves from both 2008 and 2011 were used.

spectrum was available, or the signal-to-noise ratio of the photospheric lines was insufficient for this analysis and therefore no value is given.

Co-rotation radius. A stellar rotation period independent of CoRoT light curves was measured for only one star in our sample, Mon-250, by determining the variability of the radial velocity of photospheric lines in the FLAMES spectra (Fig. 7). This method was unsuccessful for the other stars, since their radial velocities did not present significant variability within our errors. The spectroscopic period found for Mon-250 was $P_{\text {rot }}=8.6 \pm 0.5$ days, a value consistent with the periods found from its CoRoT light curves of $P_{\text {kep }}=8.3 \pm 1.0$ days in 2008 and $P_{\text {kep }}=8.9 \pm 0.5$ days in 2011. The same period of 8.6 days was found by Sousa et al. (in prep.) in both the $\mathrm{H} \alpha$ and the HeI $6678 \AA$ lines. This indicates that the structure responsible for the occultations in the CoRoT light curves is located at or near the co-rotation radius.

This was found to be true for the star AA Tau as well, when Bouvier et al. (2007) measured its rotational period using the radial velocity of the HeI $5876 \AA$ emission line and photospheric lines and found a value consistent with their photometric period. Therefore we find it reasonable to assume that the inner disk warp is located at the disk's co-rotation radius for the other AA Tau-like systems. Since we do not have an accurate measure of stellar rotation periods for the stars in our sample besides Mon-250, we use the periods determined from their CoRoT light curves in Eq. (2) to calculate their inclinations.

In order to further investigate whether these Keplerian periods can also represent the stars' rotation periods, we made a period histogram of all of the CTTS in NGC 2264 with CoRoT light curves classified as spot-like or AA Tau-like (see Fig. 13). The spot-like light curves are due to rotational modulation of stable configurations of spots on the stellar surface, and therefore these values represent the stars' rotational periods. The values from AA Tau-like light curves represent the Keplerian rotation periods of the inner disk material that occults the stellar photosphere. We see that, though the distributions are not identical, the values for AA Tau-like light curves fall within the region where rotational periods are found.
Inclination. We calculate sin $i$ of each star using Eq. (3) and taking the period found from the CoRoT light curve as $P_{\text {rot }}$, except in the case of Mon-250, for which the spectroscopic period was used. For those stars observed to be periodic in both epochs, but for which a slightly different period was found in 2008 and 2011, the value with the smallest error was used, i.e., the one whose periodogram presented a peak with smaller width at half maximum and higher intensity. The results are shown in Table 2.

It is important to note that the inclination is a very difficult parameter to determine precisely, especially when dealing with high inclinations. In these cases a relatively small error in $\sin i$ is translated into a large error in $i$. Unfortunately our errors in $\sin i$ themselves are relatively large to begin with, therefore our values for $i$ are very imprecise.

Hartmann (2001) and Rebull et al. (2002) discuss the difficulties in obtaining precise values of luminosity, temperature, and radius for TTSs because of their strong variabilities, spectrum veiling, surface spots, and uncertainties in calculating the distance. The former showed that typical errors in radius determination are on the order of $20 \%$, while the latter show errors of typically $12 \%$ for TTSs in the spectral range K5-M2. In this study we consider errors of $20 \%$ in radius.

Errors in period and $v \sin i$ vary from one star to another. For periods, we adopted the half width at half maximum of the peak in each periodogram as the uncertainty, which is typically around $5 \%$ to $7 \%$. Our errors in $v \sin i$ are typically on the order of $10-12 \%$. This leads to errors around $35 \%$ in $\sin i$, which is rather high, but consistent with other studies of TTS inclinations (e.g., Artemenko et al. 2012).

Although the actual values of inclination are imprecise, it is not difficult to distinguish between systems with low inclinations and those with medium to high inclinations, since for low inclinations errors in sin $i$ do not translate into such large errors in $i$ as with high inclinations. We see from Table 2 that the star Mon-1131 is seen at a low inclination, and we can consequently rule out the proposed occultation mechanism as the primary cause for the light curve variations. However, it is possible that there are two structures in each hemisphere, separated by $180^{\circ}$, obscuring the photosphere and making it seem as if the period were half the true value. If this were the case its inclination would be $64_{-36}^{\circ}$, though further investigation is needed to determine its true rotation period. For the time being this star has been excluded from further investigation using the occultation model, though it is studied in Stauffer et al. (2015), where different mechanisms are proposed to explain its variability.

For the other stars in our sample for which an inclination can be estimated, they tend toward high values, at least within the uncertainties (see Table 2). There are many stars for which the value of $\sin i$ found was larger than 1 . In some cases a reasonable interval of values within the error bars falls under 1 , in which case we argue that the system's inclination should be anywhere between the inverse sine of the lowest value $(\sin i-\Delta \sin i$, where $\Delta \sin i$ is the uncertainty in $\sin i)$ and $90^{\circ}$. We then consider the inclination to be the mean value in this interval (for example, a star of $\sin i=1.07 \pm 0.32$ has an inclination of $69^{\circ} \pm 21^{\circ}$, since the inverse sine of the lowest value, $\sin i=1.07-0.32=0.75$, is $48^{\circ}$ ).

For the star Mon-928, its value of $\sin i$ is much larger than 1 , even when considering the error bars. It is possible that the material occulting this star is located farther away from the star than its co-rotation radius, in which case the Keplerian period we calculated using the CoRoT light curves is larger than the stellar rotation period, resulting in a larger value of $\sin i$. It is also possible that the true period is half the value used. There is an additional, 
Table 2. Stellar parameters initially calculated for the AA Tau-like candidates.

\begin{tabular}{|c|c|c|c|c|c|c|c|c|c|c|}
\hline CSIMon ID & $\begin{array}{l}L_{\text {bol }} \\
\left(L_{\odot}\right) \\
\end{array}$ & $\begin{array}{c}M_{*} \\
\left(M_{\odot}\right) \\
\end{array}$ & $\begin{array}{c}R_{*} \\
\left(R_{\odot}\right) \\
\end{array}$ & $\begin{array}{c}P_{1} \\
\text { (days) }\end{array}$ & $\begin{array}{c}P_{2} \\
\text { (days) }\end{array}$ & $\begin{array}{c}v \sin i \\
\left(\mathrm{~km} \mathrm{~s}^{-1}\right)\end{array}$ & $\sin i$ & $\begin{array}{c}\text { Inclination } \\
\left({ }^{\circ}\right)\end{array}$ & $\begin{array}{l}R_{\mathrm{w}, 1} \\
\left(R_{*}\right) \\
\end{array}$ & $\begin{array}{l}R_{\mathrm{w}, 2} \\
\left(R_{*}\right) \\
\end{array}$ \\
\hline CSIMon-000056 & 0.77 & 1.16 & 1.52 & 5.71 & 5.86 & $11.1 \pm 2.2$ & $0.85 \pm 0.38$ & $58_{-30}^{+32}$ & 9.29 & 9.45 \\
\hline CSIMon-000250 & 1.23 & 1.35 & 1.63 & 8.32 & 8.93 & $9.8 \pm 2.2$ & $1.02 \pm 0.49$ & $62 \pm 28$ & 11.71 & 12.28 \\
\hline CSIMon-000296 & 1.57 & 1.42 & 1.71 & - & 3.91 & $17.3 \pm 2.6$ & $0.78 \pm 0.33$ & $51_{-24}^{+39}$ & 5.11 & 6.86 \\
\hline CSIMon-000297 & 1.57 & 1.42 & 1.71 & 3.16 & - & $30.8 \pm 1.8$ & $1.13 \pm 0.40$ & $69 \pm 21$ & 5.95 & - \\
\hline CSIMon-000358† & 0.22 & 0.29 & 1.39 & - & 5.86 & - & - & - & - & 6.51 \\
\hline CSIMon-000379 & 2.12 & 1.60 & 1.99 & - & 3.68 & $25.0 \pm 1.2$ & $0.91 \pm 0.30$ & $66_{-28}^{+24}$ & 5.95 & 5.89 \\
\hline CSIMon-000441† & 0.50 & 0.36 & 1.92 & 4.06 & - & - & - & - & 3.97 & - \\
\hline CSIMon-000456 & 1.58 & 1.41 & 2.00 & - & 5.03 & $19.3 \pm 1.5$ & $0.96 \pm 0.21$ & $74_{-33}^{+16}$ & - & 6.92 \\
\hline CSIMon-000498 & 3.85 & 1.90 & 2.88 & 4.23 & 4.28 & $24.1 \pm 2.4$ & $0.71 \pm 0.26$ & $45_{-19}^{+31}$ & 4.73 & 4.77 \\
\hline CSIMon-000654 & 0.47 & 0.30 & 2.03 & 4.66 & - & $18.0 \pm 3.0$ & $0.82 \pm 0.37$ & $55_{-28}^{+35}$ & 3.87 & - \\
\hline CSIMon-000660 & 1.36 & 1.40 & 1.86 & 5.25 & 5.25 & $21.4 \pm 2.5$ & $1.16 \pm 0.44$ & $68 \pm 22$ & 7.64 & 7.50 \\
\hline CSIMon-000774 & 3.10 & 1.83 & 2.49 & 3.46 & - & $30.7 \pm 1.3$ & $0.84 \pm 0.28$ & $57_{-23}^{+33}$ & 4.73 & - \\
\hline CSIMon-000811 & 1.08 & 0.91 & 1.97 & - & 7.88 & $13.5 \pm 1.7$ & $1.07 \pm 0.35$ & $64 \pm 26$ & - & 8.19 \\
\hline CSIMon-000824 & 1.96 & 1.48 & 2.23 & 7.05 & - & $14.8 \pm 1.4$ & $0.92 \pm 0.40$ & $68_{-37}^{+22}$ & 7.90 & - \\
\hline CSIMon-000928 & 0.58 & 0.63 & 1.65 & 9.92 & - & $20.1 \pm 2.8$ & $2.38 \pm 1.05$ & 90 & 10.09 & - \\
\hline CSIMon-001054 & 0.63 & 0.36 & 2.17 & - & 4.08 & $20.4 \pm 2.0$ & $0.76 \pm 0.28$ & $49_{-28}^{+41}$ & - & 3.52 \\
\hline CSIMon-001131 & 0.55 & 0.36 & 2.01 & - & 5.18 & $8.8 \pm 1.7$ & $0.45 \pm 0.21$ & $27 \pm 14$ & 4.44 & 4.46 \\
\hline CSIMon-001140 & 1.10 & 1.31 & 1.67 & 3.87 & 3.90 & $19.8 \pm 2.3$ & $0.91 \pm 0.34$ & $66_{-35}^{+24}$ & 6.79 & 6.83 \\
\hline CSIMon-001167 & 0.56 & 0.30 & 2.23 & - & 8.78 & $11.3 \pm 2.1$ & $0.88 \pm 0.42$ & $62_{-35}^{+28}$ & 5.18 & 5.37 \\
\hline CSIMon-001296 & 0.91 & 0.69 & 1.99 & - & 9.75 & $11.0 \pm 1.6$ & $1.07 \pm 0.48$ & $63 \pm 27$ & - & 8.52 \\
\hline CSIMon-001308 & 0.53 & 0.63 & 1.59 & 6.45 & 6.68 & $9.9 \pm 2.0$ & $0.82 \pm 0.37$ & $55_{-28}^{+35}$ & 7.86 & 8.04 \\
\hline CSIMon-014132† & 0.37 & 0.28 & 1.88 & - & 4.48 & - & - & - & - & 3.98 \\
\hline
\end{tabular}

Notes. $P 1$ and $P 2$ are the Keplerian periods found from the CoRoT light curves in 2008 and 2011, respectively, while $R_{\mathrm{w}, 1}$ and $R_{\mathrm{w}, 2}$ are the corotation radii found using $P 1$ and $P 2$, respectively. The stars identified with a $\dagger$ either have no observed spectrum, or the signal-to-noise ratio is too low to determine $v \sin i$, and so we cannot calculate their inclinations.

smaller, flux dip in its 2008 light curve located between the second and third larger flux dips (see Fig. 9), which justifies using a period of 4.96 days, rather than 9.92 days, though we gave preference to the latter since it coincides with the period found in the literature of $9.48 \pm 2.34$ (Lamm et al. 2005). If the period were indeed half the value we considered, this would lead to an inclination of $66^{\circ} \pm 24^{\circ}$. In either case, it is probably safe to consider that this system has a high inclination. Therefore, we used the occultation model described in Sect. 1.1 to find which interval of inclinations results in the best fit for the light curve, and consider this as the system's inclination. We did this as well for the three stars for which we have no measure of $v \sin i$, where we assume without proof that the systems are seen nearly edge-on. The new values are presented in Table 3.

For the rest of the AA Tau-like stars, we used the occultation model in a similar fashion as a fine tuning for the inclination. We noticed that in many cases we were not able to successfully reproduce the observed widths in flux dips using the whole range of values of $i$ initially calculated, unless we used other parameters that were unphysical (such as azimuthal extension $\phi_{\mathrm{w}}>360^{\circ}$ ). Therefore, we took all values of $i$ within the error bars and chose only the interval that could successfully reproduce the widths of the observed light curves with physically possible values of $\phi_{\mathrm{w}}$. This restricted the inclinations much more than the original calculation, resulting in much smaller error bars, as can be seen in Table 3. Figure 14 shows an example of the best fits for a light curve using the originally calculated value of inclination (with $\phi_{\mathrm{w}} \leq 360^{\circ}$ ) and the new value presented in Table 3. It is easy to see that this value reproduces the widths of the flux dips much more closely.


Fig. 14. Fitted light curves of the star Mon-1308 using an inclination of $55^{\circ}$ (left) and $75^{\circ}$ (right). In both cases the values of warp maximum height and azimuthal extension were set to those that best fit the width and amplitude of the minima without extrapolating to unphysical values.

Warp parameters and fitted light curves. The occultation model was used interactively to simulate the CoRoT light curves using the values given in Table 2 for stellar mass, radius, and corotation radius, with the warp maximum height and azimuthal extension as free parameters, and inclination as a semi-free parameter, constrained to values within the error bars presented in Table 2. Each minimum of each light curve was fitted independently to account for slight changes in the warp from one rotation cycle to another. Some examples of the synthetic light curves reproduced by the occultation model are shown in Fig. 15 (synthetic light curves of all other stars are shown in Fig. 23). Inclinations were determined first, as described above, 
Table 3. Occultation model parameters found for the AA Tau-like candidates.

\begin{tabular}{cccccc}
\hline \hline CSIMon ID & Epoch & $R_{\mathrm{w}}\left(R_{*}\right)$ & $h_{\max } / R_{\mathrm{w}}$ & $\phi_{\mathrm{w}}\left({ }^{\circ}\right)$ & Inclination $\left(^{\circ}\right)$ \\
\hline CSIMon-000056 & 2008 & 9.29 & $0.24-0.25( \pm 0.10)$ & $320( \pm 40)-360$ & $71 \pm 6$ \\
CSIMon-000056 & 2011 & 9.45 & $0.24-0.26( \pm 0.10)$ & $290( \pm 70)-360$ & $71 \pm 6$ \\
CSIMon-000250 & 2008 & 11.71 & $0.23-0.28( \pm 0.05)$ & $140( \pm 30)-360$ & $74 \pm 3$ \\
CSIMon-000250 & 2011 & 12.28 & $0.24-0.27( \pm 0.05)$ & $270( \pm 30)-360$ & $74 \pm 3$ \\
CSIMon-000296 & 2011 & 6.86 & $0.21-0.31( \pm 0.09)$ & $290( \pm 40)-360$ & $72 \pm 5$ \\
CSIMon-000297 & 2008 & 5.95 & $0.17-0.28( \pm 0.04)$ & $180( \pm 30)-360$ & $75 \pm 2$ \\
CSIMon-000358 & 2011 & 6.51 & $0.17-0.19( \pm 0.06)$ & $210( \pm 50)-360$ & $73 \pm 4$ \\
CSIMon-000379 & 2011 & 5.89 & $0.22-0.34( \pm 0.12)$ & $110( \pm 30)-270( \pm 90)$ & $70 \pm 7$ \\
CSIMon-000441 & 2008 & 3.97 & $0.17-0.23( \pm 0.11)$ & $160( \pm 20)-310( \pm 50)$ & $70 \pm 7$ \\
CSIMon-000456 & 2011 & 6.92 & $0.18-0.24( \pm 0.05)$ & 360 & $74 \pm 3$ \\
CSIMon-000498 & 2008 & 4.73 & $0.17-0.21( \pm 0.10)$ & $145( \pm 35)-165( \pm 15)$ & $70 \pm 6$ \\
CSIMon-000498 & 2011 & 4.77 & $0.19-0.22( \pm 0.10)$ & $130( \pm 50)-165( \pm 15)$ & $70 \pm 6$ \\
CSIMon-000654 & 2008 & 3.87 & $0.10-0.13( \pm 0.06)$ & $180( \pm 60)-310( \pm 50)$ & $73 \pm 4$ \\
CSIMon-000660 & 2008 & 7.64 & $0.25-0.33( \pm 0.04)$ & $260( \pm 20)-360$ & $74 \pm 3$ \\
CSIMon-000660 & 2011 & 7.50 & $0.27-0.29( \pm 0.05)$ & $200( \pm 30)-360$ & $74 \pm 3$ \\
CSIMon-000774 & 2008 & 4.73 & $0.15-0.21( \pm 0.03)$ & $220( \pm 40)-360$ & $75 \pm 2$ \\
CSIMon-000811 & 2011 & 8.19 & $0.26-0.28( \pm 0.08)$ & 360 & $72 \pm 5$ \\
CSIMon-000824 & 2008 & 7.90 & $0.24-0.27( \pm 0.08)$ & $260( \pm 40)-360$ & $72 \pm 5$ \\
CSIMon-000928 & 2008 & 6.36 & $0.28-0.30( \pm 0.10)$ & $120-300( \pm 60)$ & $71 \pm 6$ \\
CSIMon-001054 & 2011 & 3.52 & $0.16-0.21( \pm 0.09)$ & $270( \pm 70)-360$ & $70 \pm 5$ \\
CSIMon-001140 & 2008 & 6.79 & $0.16-0.24( \pm 0.03)$ & $190( \pm 20)-360$ & $75 \pm 2$ \\
CSIMon-001140 & 2011 & 6.83 & $0.14-0.24( \pm 0.03)$ & $190( \pm 20)-360$ & $75 \pm 2$ \\
CSIMon-001167 & 2011 & 5.37 & $0.15-0.18( \pm 0.05)$ & $330( \pm 30)-360$ & $74 \pm 3$ \\
CSIMon-001296 & 2011 & 8.52 & $0.31-0.34( \pm 0.13)$ & $260( \pm 80)-330( \pm 30)$ & $69 \pm 8$ \\
CSIMon-001308 & 2008 & 7.86 & $0.28-0.30( \pm 0.06)$ & $330( \pm 30)-360$ & $73 \pm 4$ \\
CSIMon-001308 & 2011 & 8.04 & $0.20-0.27( \pm 0.06)$ & $340( \pm 20)-360$ & $73 \pm 4$ \\
CSIMon-014132 & 2011 & 3.98 & $0.18-0.32( \pm 0.14)$ & $120( \pm 40)-290( \pm 80)$ & $68 \pm 9$ \\
\hline
\end{tabular}

Notes. $R_{\mathrm{w}}$ is the radius where the warp is located, assumed to be at the co-rotation radius (i.e., $R_{\mathrm{w}}=R_{\mathrm{co}}$ ); $h_{\max }$ is the maximum warp height found for each flux dip, expressed in stellar radii $R_{*}$; and $\phi_{\mathrm{w}}$ is the azimuthal extension of the warp found for each flux dip. The inclinations shown here were constrained using the occultation model.

by verifying for which values we were able to reproduce the width of each minima without having to resort to unphysical values of azimuthal extension. We used a maximum inclination of $77^{\circ}$, since for inclinations larger than this the outer flared disk would overshadow the inner disk region ${ }^{4}$, making it impossible to observe the stellar photosphere (Bertout 2000). Error bars in warp maximum height and azimuthal extension were calculated by modeling the minima with the value of inclination fixed at its lowest and highest possible values, and finding the best fit in each case.

Table 3 shows the final values obtained for the model parameters. Since they vary from one rotation cycle to another, we present the intervals of the values found for each light curve. Most of the values found for the warp's maximum height and azimuthal extension are similar to those found for AA Tau. The average ratio between the warp's maximum height and the radius at which it is located is $h_{\max } / R_{\mathrm{w}}=0.23$, though individual values range from 0.10 to 0.34 (for comparison we note that the value used for AA Tau in Bouvier et al. 1999 was of $\left.h_{\max } / R_{\mathrm{w}}=0.30\right)$. The average value of the warp's azimuthal extension is $\phi_{c}=300^{\circ}$, but is often a full $360^{\circ}$, the same as

\footnotetext{
4 This effect may not be as significant for sources that have anemic disks, since they may possess flatter outer disks. However, this would only be relevant for the star Mon-314, which has aperiodic photometric behavior and therefore is not analyzed using the occultation model at any rate.
}

AA Tau, and can be as low as $110^{\circ}$. Inclinations generally need to be greater than $\sim 65^{\circ}$ for the occultation model to successfully reproduce the widths of the minima, but in some cases may be as low as $60^{\circ}$ for relatively large warp heights and shallow flux dips.

We see that the warp maximum height should vary between rotation cycles by on average $11 \%$, and at times by up to $57 \%$, to account for the variations in amplitude of the light curves. The azimuthal extension of the warp should vary by on average $17 \%$, and up to $65 \%$, between rotation cycles to account for the variations in width of the minima present in the CoRoT light curves. If the photometric variability of these stars is truly due to occultations by an inner disk warp caused by the interaction between the magnetosphere and the inner disk region, as we propose, then these values show how dynamic this interaction is, as has been predicted by MHD models.

\subsection{Color-magnitude diagrams and color variation}

We constructed color magnitude diagrams of the stars in our sample (both those that present AA Tau-like light curves and those that present aperiodic extinction dominated light curves), to search for trends characteristic of extinction. We used data taken simultaneously with CoRoT and Spitzer, plotting CoRoT magnitude against CoRoT magnitude minus Spitzer IRAC $_{4.5} \mu \mathrm{m}$ magnitude. It is not possible to use this method to clearly 



Fig. 15. Examples of simulated light curves of AA Tau-like stars (red) plotted over CoRoT light curves (black).

distinguish if the photometric variability is due to extinction or a configuration of spots on the surface, but if we assume that extinction is the main source of the flux dips present in both the CoRoT and IRAC light curves, then we can estimate extinction laws from the slopes of the distributions (Fig. 16).

In order to be sure that we are analyzing parts of the light curve where the same phenomenon is responsible for the variability in the optical and infrared bands, we calculated slopes using linear regression only for the parts of the data where the CoRoT and IRAC light curves correlate reasonably well. We also separated these parts in order to calculate slopes for different flux dips and different parts of the flux dips, as shown in Fig. 16, in order to verify if different extinction laws could be found for different parts of the disk warp. We find that entrances into and exits from the occultations show the same slopes, within the uncertainties. In one case, Mon-456 (see left panels of Fig. 16), slightly different slopes were found during different flux dips. This might indicate a small variability in the grain distribution of the inner disk warp during rotation cycles, or it could be due to other factors, such as variable disk emission, interfering with the IRAC light curves.

We were able to estimate extinction laws for ten of the stars in our sample using this method, finding values between those consistent with ISM $\left(A_{4.5} \mu \mathrm{m} / A_{R} \sim 0.05\right)$ and up to $A_{4.5 \mu \mathrm{m}} / A_{R} \sim$ $0.29 \pm 0.04$. Slopes of values less than 1 were not considered, since extinction laws derived would be unphysical. In these cases, other factors, such as variable disk emission in $4.5 \mu \mathrm{m}$ or a configuration of hot or cold spots on the stellar surface, could be strongly influencing the distribution of points in the color magnitude diagrams. It is possible that variable disk emission is also influencing our derived extinction laws, causing us to find lower slopes and therefore lower extinction laws than were estimated in Sect. 3.4. More accurate estimates will require a more extensive study of these and other color magnitude diagrams, which will be treated in a future paper.

To check for color variation in the periodic and quasiperiodic stars in our sample, we compared CoRoT and I-band light curves with $u-r$ for the stars whose light curves were classified as AA Tau-like or AA Tau candidates in 2011. We plotted these light curves in phase, using the periods determined from the CoRoT data (Table 1). The same initial date was taken for all three plots for easy comparison. Though the CFHT data were not taken simultaneously with CoRoT, by folding the light curves in phase, using the same initial date and period, we can see how the color varies during the flux dips. For most stars, we see a reddening at phase 0.5 during the eclipses, as is to be expected from an extinction event.

For four stars (Mon-250, Mon-358, Mon-456, and Mon-1054), we can observe a bluing at phase 0.5 for one or two rotation cycles, while the other cycles either maintain relatively constant color or become slightly redder. This effect was observed in AA Tau (Bouvier et al. 2003), which showed little color variation except during some minima, when it became bluer. This was attributed to the appearance of part of the hot spot associated with the accretion shocks on the stellar surface, during eclipses from the inner disk warp. This shows very strong evidence that these four stars are undergoing the same physical processes as AA Tau did during its stable accretion phase.

Three stars undergo an interesting phenomenon where they become slightly bluer during the beginning of the eclipses and then become redder. This could also be evidence that the hot spot associated with the accretion shock is partially showing, but in this case it is soon occulted by the inner disk warp. These stars are Mon-1140, Mon-1308, and Mon-1167. For two stars, Mon-56 and Mon-14132, we see little or no change in color. Figure 17 shows some of these light curves and $u-r$ color diagrams folded in phase. The others are shown in Fig. 24.

\subsection{Other characteristics of AA Tau-like stars}

Figure 18, top left panel, shows a mass histogram of AA Tau-like stars (red) and stars with aperiodic extinction dominated light curves (purple), plotted over a histogram of all CTTS in our 



Fig. 16. Top: CoRoT light curve of the stars Mon-456, Mon-660, and Mon-1054. Bottom: CoRoT vs. CoRoT-IRAC $4.5 \mu \mathrm{m}$ color magnitude diagrams of the same stars. The slopes were calculated for various parts of the light curves where CoRoT and Spitzer IRAC data best correlate. Different colors show different parts of the light curves for which slopes were calculated.

study of NGC 2264 that present CoRoT light curves from either observing run. While the CTTS show a tendency toward lower masses, the number of stars declining rapidly for larger masses, the AA Tau-like stars are equally present in the whole range of masses considered. Therefore, though there are AA Tau-like stars of all masses in the CTTS range, the ratio of these stars to the total number of stars in that mass bin increases with increasing mass, meaning they are relatively more common among higher mass stars than lower mass ones (top right diagram in Fig. 18). Stars that present AA Tau-like photometric behavior in either 2008 or 2011 were included in the AA Tau category.

Inclination is a major factor in whether we observe AA Tau-like events or not; therefore, we know that the observed AA Tau-like stars are only a fraction of the total number of stars that present an inner disk warp. For stars of $M \lesssim 0.7 M_{\odot}$, fewer than $10 \%$ are observed as AA Tau-like. If we consider that it is possible to view the occultations at angles between $\sim 60^{\circ}$ and $77^{\circ}$, as was shown using the occultation model, then only at most $50 \%$ of stars in this mass range possess an inner disk warp with two major accretion funnels. For stars of $M \gtrsim 0.7 M_{\odot}$, about $35 \%$ show AA Tau-like behavior, meaning that if we assume random inclinations, all of these stars likely possess an inner disk warp during some part of their evolution.

The fraction of stars that present AA Tau-like photometric behavior is quite large as we approach even higher masses $\left(M \gtrsim 1.0 M_{\odot}\right)$, much larger than we would expect if all CTTS observed by CoRoT possessed an inner disk warp, given that when we consider random inclinations we should only see $\sim 20 \%$ of AA Tau-like systems. This is possibly due to a selection bias, since the CoRoT targets are not selected randomly, but based on specific criteria, which included many systems with known periodicity, and in 2011 included systems previously classified as AA Tau-like. Therefore, to compare with a less biased sample, we plotted a histogram of the number of AA Tau-like systems observed by CoRoT compared to the total number of
CTTS observed by the CFHT MegaCam (presented in Venuti et al. 2014), including those that were and were not observed by CoRoT. This plot, shown in the bottom panel of Fig. 18, may also have a biased tendency, since it is possible that some of the systems not observed by CoRoT also present AA Tau-like variability that would be unaccounted for. Even so, we see that slightly over $20 \%$ of stars with mass $M \gtrsim 1.0 M_{\odot}$ present this type of variability. Extrapolating this amount to the systems where the inclinations prevent us from observing this tendency, we still conclude that nearly all of the systems with mass $1.0 M_{\odot} \lesssim M \lesssim 2.0 M_{\odot}$ and most ( 75\%) of the systems with mass $0.7 M_{\odot} \lesssim M \lesssim 1.0 M_{\odot}$ should present an inner disk warp, while for systems of $M \lesssim 0.7 M_{\odot}$ this fraction would be of only $\sim 25 \%$.

This may be due to different stellar magnetic field configurations. The dipolar component of the magnetic field is responsible for the interaction with the inner disk, and a stronger dipolar component leads to a larger truncation radius. As was discussed in Sect. 3.3, a larger truncation radius tends to favor a stable accretion regime. Gregory et al. (2012) found that the magnetic field configuration of a star depends strongly on its internal structure, and that for intermediate to high mass $\mathrm{T}$ Tauri Stars $\left(0.5 M_{\odot} \lesssim M_{*} \lesssim 2.0 M_{\odot}\right)$, those that are fully convective, or have as yet only developed a small radiative core, tend to have strong dipoles. At the estimated age of 2-3 Myr of NGC 2264, Gregory et al. (2012) show that stars in this mass range have not yet developed a significant radiative core. They should therefore possess strong dipolar components in their magnetic fields.

There is still very little information available on magnetic fields of T Tauri stars of smaller mass $\left(M_{*} \lesssim 0.5 M_{\odot}\right)$, because of observational constraints. At least one T Tauri star in this mass range, V2247 Oph, has had its magnetic field topology measured and was found to have a weak dipolar component (Donati et al. 2010). Therefore, it is possible that among fully convective stars, a significant difference in magnetic field configurations 

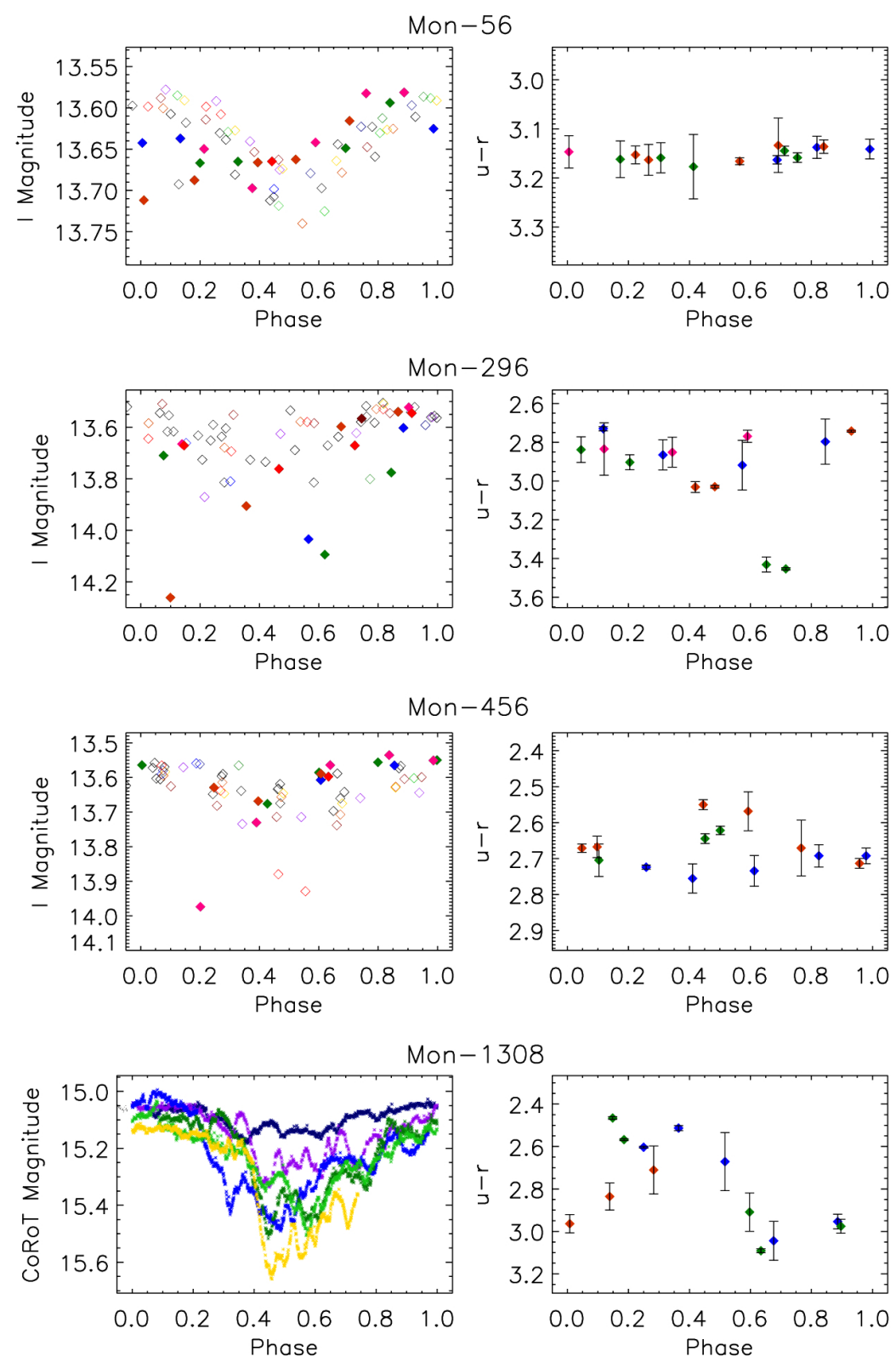

Fig. 17. Examples of $I$-band and $u-r$ light curves folded in phase. The colors used for $u-r$ represent the same rotation cycle as the filled diamonds of the same color in $I$. Mon-56 shows no measurable variability in $u-r$; Mon-296 shows reddening events in $u-r$ during its eclipses in the $I$-band; Mon-456 shows a slight reddening during one of its $I$-band minima (blue diamonds) and bluing events during the other $I$-band minima (orange and green diamonds); and Mon-1308 shows a bluing, followed by a reddening event during its minima in the CoRoT light curve. It was not possible to identify flux dips in the phase-folded $I$-band light curve of Mon-1308 because of insufficient cadence, so the phase-folded CoRoT light curve is shown instead. The same period and initial date were used for CoRoT and $u-r$, though in this case colors do not represent the same phase in both plots.

may exist among these two mass ranges. Morin et al. (2010) showed that among main sequence stars, those of very low mass show magnetic field topologies that differ considerably from fully convective stars of somewhat higher mass. While the latter generally possess magnetic fields with strong dipoles, main sequence stars of very low mass $\left(M_{*} \lesssim 0.2 M_{\odot}\right)$ show a number of different magnetic topologies, ranging from very strong, axisymmetric, nearly dipolar fields to weaker fields with a strong non-axisymmetric component, and a strong toroidal component. This supports the idea that a different magnetic field regime may dominate among stars of lower mass.

However, our results alone are insufficient to conclude that a significant difference exists among magnetic field configurations in these two mass ranges. There are other factors that may influence our higher detection of AA Tau-like systems among those of somewhat higher mass. For instance, mass accretion rates tend to decrease toward lower masses (Venuti et al. 2014), which would increase the truncation radius at this mass range. This would favor a stable accretion mechanism, but could also lead to the disk warp being located farther away from the co-rotation radius than we have assumed. If they are located significantly farther away, we would need higher inclinations to observe the eclipses in these systems, making it more difficult to do so.

It is also possible that this effect is simply the result of an observational bias. Stars of lower mass are generally fainter than those of higher mass, and the signal from the CoRoT 



Fig. 18. Top left: mass histogram of all CTTS with CoRoT light curves (black), stars with AA Tau-like light curves (red), and stars whose CoRoT light curves are dominated by aperiodic extinction events (purple). Top right: number of AA Tau-like stars (red) and aperiodic extinction stars (purple), divided by the total number of CTTS observed by CoRoT in that mass bin. Stars of both CoRoT observing runs were used, and any star that presented AA Tau-like photometric behavior in either run was included in the AA Tau category. Bottom: number of AA Tau-like stars (red) and aperiodic extinction stars (purple), divided by the total number of CTTS in the sample of Venuti et al. (2014), including those that were not observed by CoRoT.

observations could be too low among stars below a certain mass limit to allow an accurate classification of AA Tau-like light curves.

\section{Discussion}

The occultation model initially proposed for the star AA Tau appears to be adequate at reproducing the general aspects of the flux dips of the stars listed in Table 3. It is capable of reproducing their widths and amplitudes using values of inclination, warp maximum height, and azimuthal extension that are consistent with those found for AA Tau itself, with only a few stars showing values of warp height and azimuthal extension that are significantly lower.

We note that for stars whose light curves are classified as AA Tau-like in both CoRoT observing runs (2008 and 2011), the values found for warp maximum height and azimuthal extension in one epoch are always within the same range as those found in the other. This means that, although the occulting structure should be variable on a timescale of days to weeks because of the dynamic interactions, it should be stable enough to persist on a timescale of a few years. We also note that, for these stars, the periods found in each observing run are very similar to each other. Most are equal within their uncertainties. In all of these cases the radius where the occulting structure should be located differs by less than $4 \%$ from one epoch to the other, and most by less than $2 \%$, which is within their respective uncertainties.
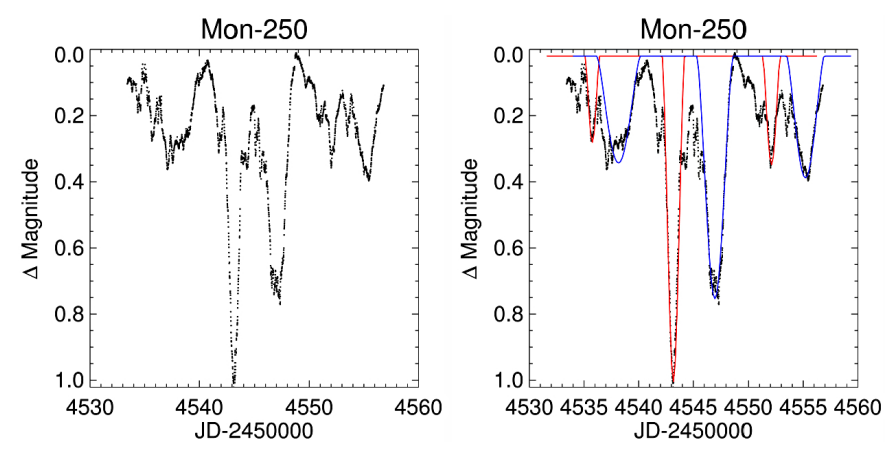

Fig. 19. Left: 2008 CoRoT light curve of Mon-250. Right: synthetic light curves generated with the occultation model for two separate accretion funnels (blue and red) overplotted on the 2008 CoRoT light curve of Mon-250.

It is possible that the structures move somewhat in radius over timescales of a few years, but they should do so by very little.

Though it can reproduce the widths and amplitudes of light curve minima, the occultation model is unable to account for the exact shape of the flux dips, which are much more complex than the nearly Gaussian shapes that it reproduces. This model is based on a symmetric warp of homogeneous optical thickness, which likely does not reflect the reality of these systems. For AA Tau, short term brightening episodes were seen to occur during the occultation events, and were attributed to inhomogeneities of reduced optical thickness in the occulting material (Bouvier et al. 1999). We observe these brightening episodes in many of the light curves in our sample. In most cases the light curve minima present significant structure, which could be due to these inhomogeneities, to the existence of a configuration of hot spots visible during the occultations, or to non-steady accretion within the funnel flows, which would lead to variable emission during the flux dips.

In some cases we observe two minima where we would expect to see one, such as in the 2008 CoRoT light curve of the star Mon-250 (left panel of Fig. 19). We know from its 2011 CoRoT light curve and spectra that this star's rotation period is on the order of 8.6 days (Fig. 7); therefore, the 2008 light curve spans only three rotation cycles, though we see more than three flux dips. We can speculate that the two middle dips are part of one structure, as the last two dips would be, and that there is significant emission from the hot spot associated with the accretion shock superimposed onto the extinction from the base of the accretion funnel, or simply that the warp was very inhomogeneous. Unfortunately, there is no color information simultaneous with this light curve to verify these scenarios. However, the occultation model is incapable of reproducing one flux dip that would encompass both middle dips seen in the light curve using physically plausible parameters. We are able to reproduce each dip separately, however, if we consider that there are two accretion funnels, separated by $160^{\circ}$, i.e., nearly opposite each other (right panel of Fig. 19). We propose that it is possible for the main accretion funnel to at times give way to two accretion funnels as a result of an increase in instabilities.

This same behavior was observed in AA Tau in 1999, when its quasi-periodic flux dips gave way to two flux dips per stellar rotation (see Fig. 15 in Bouvier et al. 2007). We may also be seeing this in the star Mon-1054, whose veiling features support the AA Tau-like scenario as the primary cause for its photometric variability (see Sect. 3.2). The left panel of Fig. 20 shows its 2011 CoRoT light curve. Arrows point to moments where we observe two flux dips when we would expect to see only one in a 



Fig. 20. Left: 2011 CoRoT light curve of Mon-1054. Arrows show moments when two flux dips are present where we would expect to see only one in a typical AA Tau-like scenario. Right: simulated light curve (red) overplotted on the 2011 CoRoT light curve of Mon-379 (black).

typical AA Tau-like scenario. The occultation model is capable of reproducing some of these double dips with only one accretion funnel, assuming that another mechanism is responsible for the emission within the dip, but it is not able to do so for the fifth double dip indicated in the left panel of Fig. 20. In this case, only two funnels (separated by $160^{\circ}$ of similar height and azimuthal extension) can successfully reproduce both minima in the light curve. This may be an indication that this star is undergoing a transition between a stable and an unstable accretion regime, or that it is in an intermediate state of some instability.

This may be the case as well for the star Mon-379 during the 2011 CoRoT observations (Fig. 20, right panel), where we can observe periodic AA Tau-like dips that are easily reproduced by the occultation model accompanied by other extinction events. These additional extinction events may be due to random accretion streams characteristic of an unstable accretion regime, as was discussed in Sect. 3.3, that coexist with the main stable accretion funnel. We also note that for this particular star some of the dips are quite narrow and the best fit found with the occultation model has azimuthal extension as low as $110^{\circ}$, unlike the majority of other stars where this value is between $260^{\circ}$ and $360^{\circ}$. This may also be an indication of this star's declining stability.

The phenomenon observed in the aperiodic light curves is similar to those observed in UX Ori-type objects, which exhibit deep, non-periodic algol-like minima caused by extinction from circumstellar material in the observer's line of sight (see the review by Grinin 2000). Both our aperiodic stars and the UX Ori-type objects show evidence of being seen at high inclinations, which is favorable for circumstellar material located near the disk plane to intersect our line of sight. UX Ori flux dips typically present larger amplitudes and longer duration than the stars in our sample, probably because of larger scale disk instabilities. They are also much more common among Herbig AeBe stars, though a few CTTS have been classified as UXors (Herbst \& Shevchenko 1999; Grinin 2000). It is possible that some of the stars in our sample with aperiodic behavior could be considered UXors, though a study of color variability and polarization would be required to determine this.

\section{Conclusions}

CTTS that present extinction dominated light curves, such as the quasi-periodic AA Tau-like behavior or aperiodic extinction events, are common in NGC 2264. Of 159 cluster members that show signs of accretion, 33 present one of these two behaviors, i.e., $(21 \pm 4) \%$. These objects give us insight into the phenomena that take place in the inner region of circumstellar disks, a region that is otherwise difficult to study because of its proximity to the star and to instrumental limitations.

Extinction laws that include dust grains considerably larger than those found typically for the interstellar medium are necessary to account for the ratios between amplitudes in optical and IR light curves. This suggests that grain growth has occurred in these disks.

A model proposed to explain the light curve of the CTTS AA Tau, in which at the base of an accretion column there is an optically thick warp in the inner circumstellar disk region that occults the stellar photosphere periodically, was tested on 21 CTTS in NGC 2264. This occultation model was shown to be successful at reproducing the widths and amplitudes of AA Taulike flux dips in the light curves of most of these stars, using warp parameters that are similar to those used for AA Tau. Typical ratios between this warp's maximum height and the radius at which it is located were found to be between $h_{\max } / R_{\mathrm{W}}=0.2$ and $h_{\mathrm{max}} / R_{\mathrm{w}}=0.3$. The warp's height and azimuthal extension were shown to be variable on a timescale of days, presenting typical variations of $10-20 \%$ between rotation cycles, though there is strong evidence that the occulting structure can exist over timescales of a few years. We have shown that, when this is the case, the warp maintains similar parameters during that time and remains at about the same radial distance.

For the few cases where the occultation model is unable to reproduce an AA Tau-like light curve using a classical inner disk warp, we have proposed a scenario where more than one accretion column exists in each hemisphere, and dust is lifted above the disk plane at other locations around the disk, all of which occult the star. This can account for the multiple flux dips per stellar rotation seen in the light curves. In many cases, even when a single accretion column in the visible hemisphere is able to account for the main features of an AA Tau-like light curve, we observe various narrow, shallow dips alongside the deeper, broader flux dips attributed to occultation by the warp associated with the main accretion column. We attribute these traits to the existence of secondary accretion streams that coexist with the main accretion funnel flows.

We ascribe AA Tau-like light curves to a stable accretion scenario, and light curves that present aperiodic extinction events to an unstable accretion scenario, and have shown that a star may suffer a transition from one to the other in the few years that separate our two CoRoT observing runs. For two stars with aperiodic extinction dominated light curves, we find evidence of hot spots associated with occultations, through an increase in veiling during light curve minima. We have also shown that AA Tau-like light curves are more common among stars of intermediate to high mass within the CTTS range $\left(0.7 M_{\odot} \lesssim M_{*} \lesssim 2.0 M_{\odot}\right)$ than among those of lower mass $\left(M_{*} \lesssim 0.7 M_{\odot}\right)$, an aspect we believe may be linked to stellar magnetic field configurations.

We have shown that all but one star in our initial sample of possible AA Tau-like systems (Mon-1131, which is seen at a low inclination) fit very well within the AA Tau-like scenario. For nine of these stars we see clear evidence of a hot spot appearing simultaneously, or nearly so, with the occultation, either through an increase in veiling in the spectra or through a bluing effect observed in the color photometry. This strongly supports the idea that the flux dips observed in the CoRoT light curves are due to occultation by an inner disk warp that is associated with stable accretion funnel flows. We can therefore state with reasonable confidence that these are AA Tau-like systems. 
Acknowledgements. The authors thank the referee V. Grinin for his contribution to the discussion. This work is based on data collected by the CoRoT satellite, and in part on observations made with the Spitzer Space Telescope, operated by the Jet Propulsion Laboratory, California Institute of Technology, under a contract with NASA. Support for this work was provided by NASA through an award issued by JPL/Caltech. PTM, SHPA, MMG, APS and NNJF acknowledge funding support from CAPES, CNPq, FAPEMIG, and Cofecub. J.B. acknowledges funding support from Cofecub, CNES, and the grant ANR 2011 Blanc SIMI5-6 02001.

\section{References}

Alencar, S. H. P., Teixeira, P. S., Guimarães, M. M., et al. 2010, A\&A, 519, A88 Artemenko, S. A., Grankin, K. N., \& Petrov, P. P. 2012, Astron. Lett., 38, 783

Baglin, A., Auvergne, M., Barge, P., et al. 2006, in ESA SP 1306, eds. M. Fridlund, A. Baglin, J. Lochard, \& L. Conroy, 33

Bertout, C. 2000, A\&A, 363, 984

Bessolaz, N., Zanni, C., Ferreira, J., Keppens, R., \& Bouvier, J. 2008, A\&A, 478,155

Boulade, O., Charlot, X., Abbon, P., et al. 2003, in Instrument Design and Performance for Optical/Infrared Ground-based Telescopes, eds. M. Iye \& A. F. M. Moorwood, SPIE Conf. Ser., 4841, 72

Bouvier, J., Chelli, A., Allain, S., et al. 1999, A\&A, 349, 619

Bouvier, J., Grankin, K. N., Alencar, S. H. P., et al. 2003, A\&A, 409, 169

Bouvier, J., Alencar, S. H. P., Boutelier, T., et al. 2007, A\&A, 463, 1017

Bouvier, J., Grankin, K., Ellerbroek, L. E., Bouy, H., \& Barrado, D. 2013, A\&A, 557, A77

Cardelli, J. A., Clayton, G. C., \& Mathis, J. S. 1989, ApJ, 345, 245

Cody, A. M., Stauffer, J., Baglin, A., et al. 2014, AJ, 147, 82

Dahm, S. E. 2008, in Handbook of Star Forming Regions, Vol. I., ed. B. Reipurth, 966

Donati, J.-F., Skelly, M. B., Bouvier, J., et al. 2010, MNRAS, 402, 1426

Donati, J.-F., Bouvier, J., Walter, F. M., et al. 2011, MNRAS, 412, 2454

Fazio, G. G., Hora, J. L., Allen, L. E., et al. 2004, ApJS, 154, 10

Flaccomio, E., Micela, G., \& Sciortino, S. 2006, A\&A, 455, 903

Flaherty, K. M., \& Muzerolle, J. 2010, ApJ, 719, 1733

Fonseca, N. N. J., Alencar, S. H. P., Bouvier, J., Favata, F., \& Flaccomio, E. 2014, A\&A, 567, A39

Fưrész, G., Hartmann, L. W., Szentgyorgyi, A. H., et al. 2006, ApJ, 648, 1090

Gregory, S. G., Donati, J.-F., Morin, J., et al. 2012, ApJ, 755, 97

Grinin, V. P. 2000, in Disks, Planetesimals, and Planets, ed. G. Garzón, C. Eiroa, D. de Winter, \& T. J. Mahoney,ASP Conf. Ser., 219, 216

Hartmann, L. 2001, AJ, 121, 1030

Hartmann, L., Hewett, R., \& Calvet, N. 1994, ApJ, 426, 669

Herbst, W., \& Shevchenko, V. S. 1999, AJ, 118, 1043

Hirose, S., \& Turner, N. J. 2011, ApJ, 732, L30

Johns-Krull, C. M., Valenti, J. A., Piskunov, N. E., Saar, S. H., \& Hatzes, A. P. 2001, in Magnetic Fields Across the Hertzsprung-Russell Diagram, eds.

G. Mathys, S. K. Solanki, \& D. T. Wickramasinghe, ASP Conf. Ser., 248, 527
Kochukhov, O. P. 2007, in Physics of Magnetic Stars, eds. I. I. Romanyuk, D. O. Kudryavtsev, O. M. Neizvestnaya, \& V. M. Shapoval, 109

Kulkarni, A. K., \& Romanova, M. M. 2009, MNRAS, 398, 701

Kupka, F., Piskunov, N., Ryabchikova, T. A., Stempels, H. C., \& Weiss, W. W. 1999, A\&AS, 138, 119

Kupka, F. G., Ryabchikova, T. A., Piskunov, N. E., Stempels, H. C., \& Weiss, W. W. 2000, Baltic Astron., 9, 590

Kurosawa, R., \& Romanova, M. M. 2013, MNRAS, 431, 2673

Kurosawa, R., Harries, T. J., \& Symington, N. H. 2006, MNRAS, 370, 580

Lada, C. J., Muench, A. A., Luhman, K. L., et al. 2006, AJ, 131, 1574

Lamm, M. H., Bailer-Jones, C. A. L., Mundt, R., Herbst, W., \& Scholz, A. 2004, A\&A, 417, 557

Lamm, M. H., Mundt, R., Bailer-Jones, C. A. L., \& Herbst, W. 2005, A\&A, 430, 1005

Lejeune, T., Cuisinier, F., \& Buser, R. 1997, A\&AS, 125, 229

Lima, G. H. R. A., Alencar, S. H. P., Calvet, N., Hartmann, L., \& Muzerolle, J. 2010, A\&A, 522, A104

Morin, J., Donati, J.-F., Petit, P., et al. 2010, MNRAS, 407, 2269

Muzerolle, J., Calvet, N., \& Hartmann, L. 2001, ApJ, 550, 944

Pasquini, L., Avila, G., Blecha, A., et al. 2002, The Messenger, 110, 1

Piskunov, N. E., Kupka, F., Ryabchikova, T. A., Weiss, W. W., \& Jeffery, C. S. 1995, A\&AS, 112, 525

Preibisch, T., Kim, Y.-C., Favata, F., et al. 2005, ApJS, 160, 401

Ramírez, S. V., Rebull, L., Stauffer, J., et al. 2004, AJ, 127, 2659

Rebull, L. M., Makidon, R. B., Strom, S. E., et al. 2002, AJ, 123, 1528

Rebull, L. M., Cody, A. M., Covey, K. R., et al. 2014, AJ, 148, 92

Romanova, M. M., Ustyugova, G. V., Koldoba, A. V., \& Lovelace, R. V. E. 2009, MNRAS, 399, 1802

Romanova, M. M., Ustyugova, G. V., Koldoba, A. V., \& Lovelace, R. V. E. 2013, MNRAS, 430, 699

Romanova, M. M., Lovelace, R. V. E., Bachetti, M., et al. 2014, in Eur. Phys. J. Web Conf., 64, 5001

Ryabchikova, T. A., Piskunov, N. E., Kupka, F., \& Weiss, W. W. 1997, Baltic Astron., 6, 244

Samadi, R., Fialho, F., Costa, J. E. S., et al. 2006, in ESA SP 1306, eds. M. Fridlund, A. Baglin, J. Lochard, \& L. Conroy, 317

Shu, F., Najita, J., Ostriker, E., et al. 1994, ApJ, 429, 781

Siess, L., Dufour, E., \& Forestini, M. 2000, A\&A, 358, 593

Stauffer, J., Cody, A. M., Baglin, A., et al. 2014, AJ, 147, 83

Stauffer, J., Cody, A. M., McGinnis, P., et al. 2015, AJ, accepted

Teixeira, P. S., Lada, C. J., Marengo, M., \& Lada, E. A. 2012, A\&A, 540, A83

Terquem, C., \& Papaloizou, J. C. B. 2000, A\&A, 360, 1031

Turner, N. J., Carballido, A., \& Sano, T. 2010, ApJ, 708, 188

Valenti, J. A., \& Piskunov, N. 1996, A\&AS, 118, 595

Venuti, L., Bouvier, J., Flaccomio, E., et al. 2014, A\&A, 570, A82

White, R. J., \& Basri, G. 2003, ApJ, 582, 1109

Whitney, B. A., Robitaille, T. P., Bjorkman, J. E., et al. 2013, ApJS, 207, 30

Young, A. 1978, PASP, 90, 144

Zanni, C., \& Ferreira, J. 2013, A\&A, 550, A99

Pages 23 to 27 are available in the electronic edition of the journal at http://www. aanda.org 
P. T. McGinnis et al.: Probing the inner disks of AA Tau-like systems in NGC 2264


Fig. 21. LiIEW vs. I magnitude or CoRoT counts.
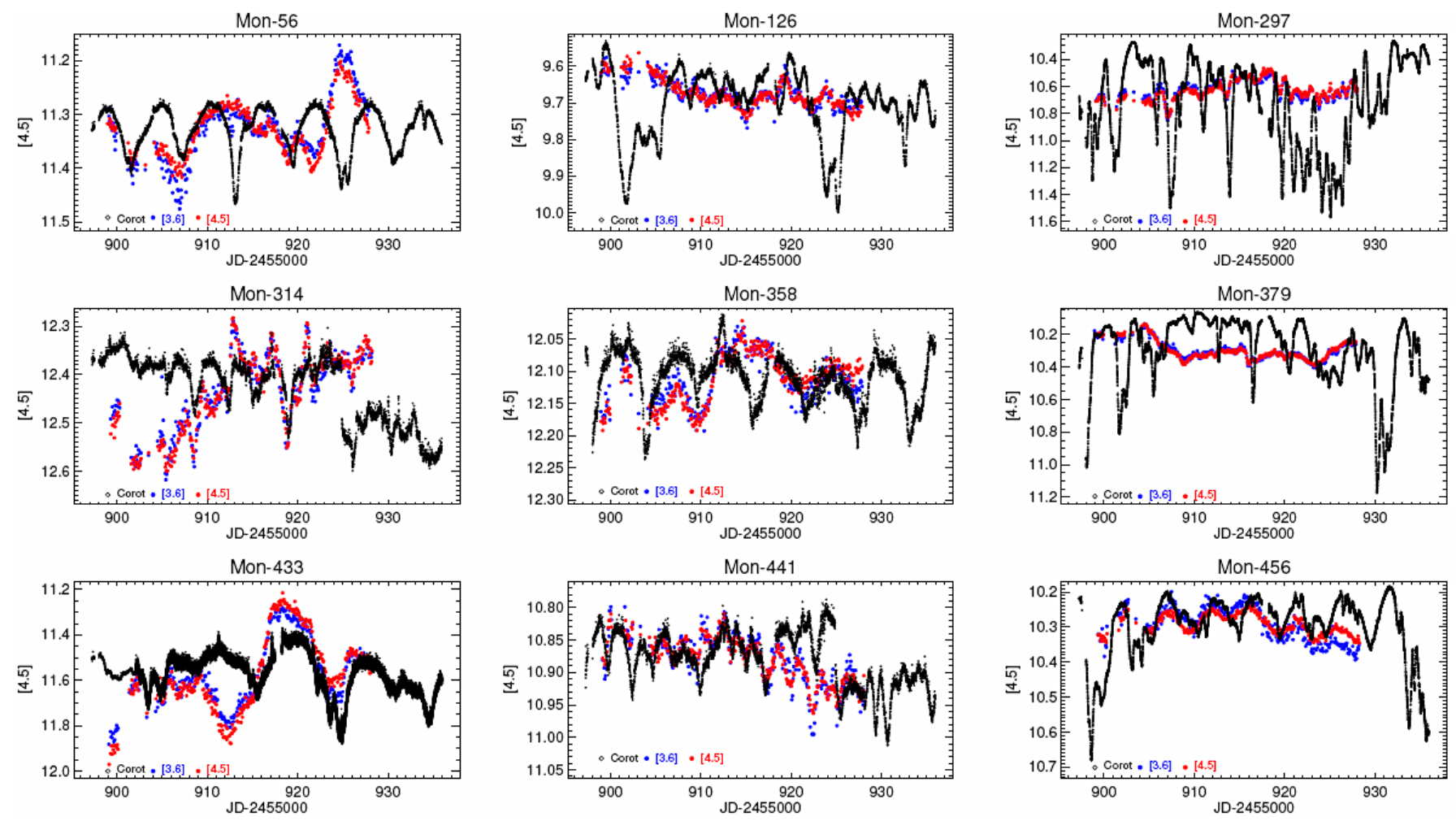

Fig. 22. Spitzer IRAC $3.6 \mu \mathrm{m}$ and $4.5 \mu \mathrm{m}$ light curves (blue and red filled circles, respectively) overplotted on CoRoT light curves (black circles). The CoRoT and IRAC $3.6 \mu \mathrm{m}$ light curves were shifted in magnitude for easier comparison. 



Fig. 22. continued. 
P. T. McGinnis et al.: Probing the inner disks of AA Tau-like systems in NGC 2264


Fig. 23. Simulated light curves of AA Tau-like stars (red) plotted over CoRoT light curves (black). 

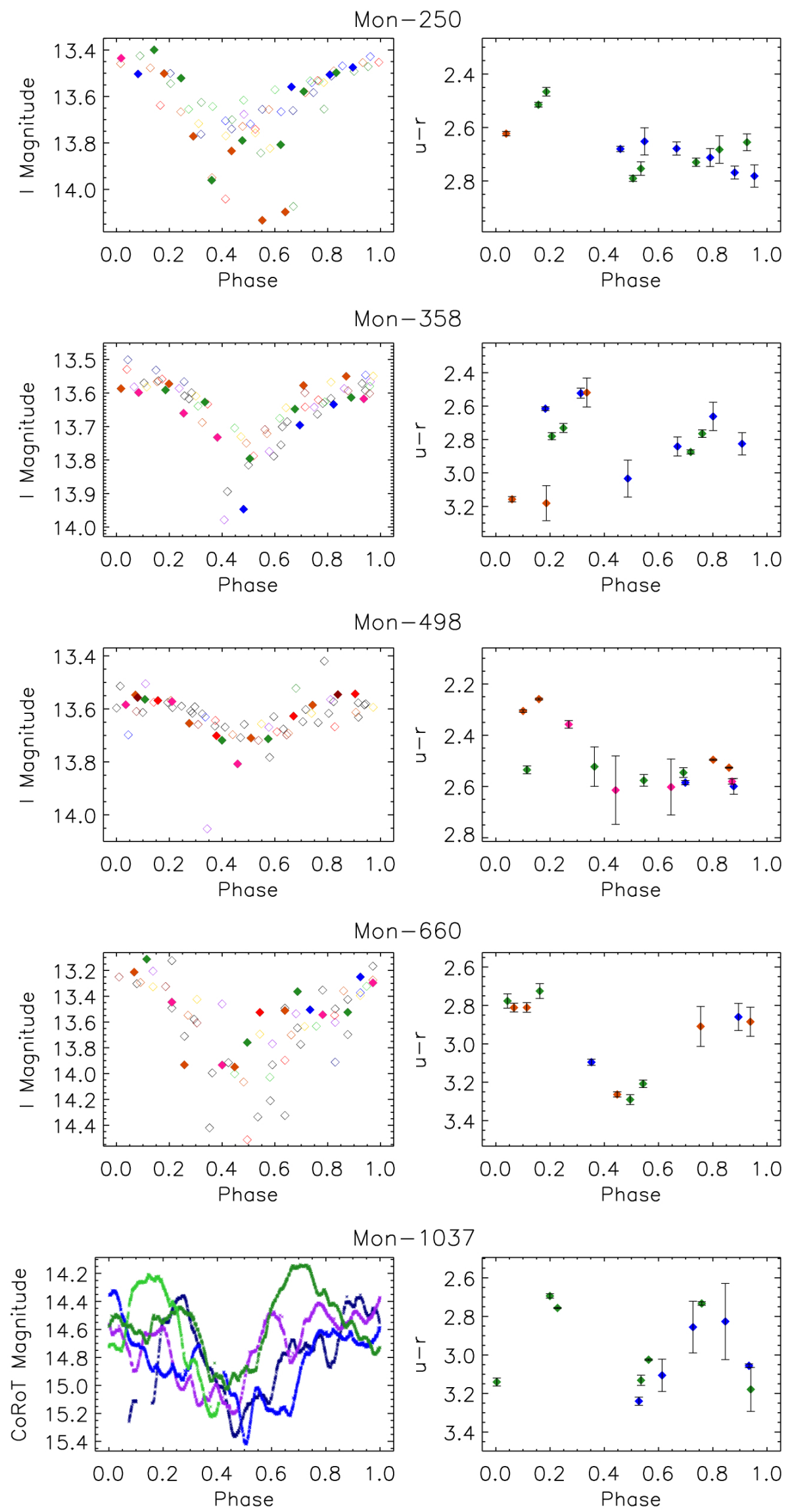

Fig. 24. $I$-band or CoRoT light curves and $u-r$ color diagrams folded in phase. 
P. T. McGinnis et al.: Probing the inner disks of AA Tau-like systems in NGC 2264
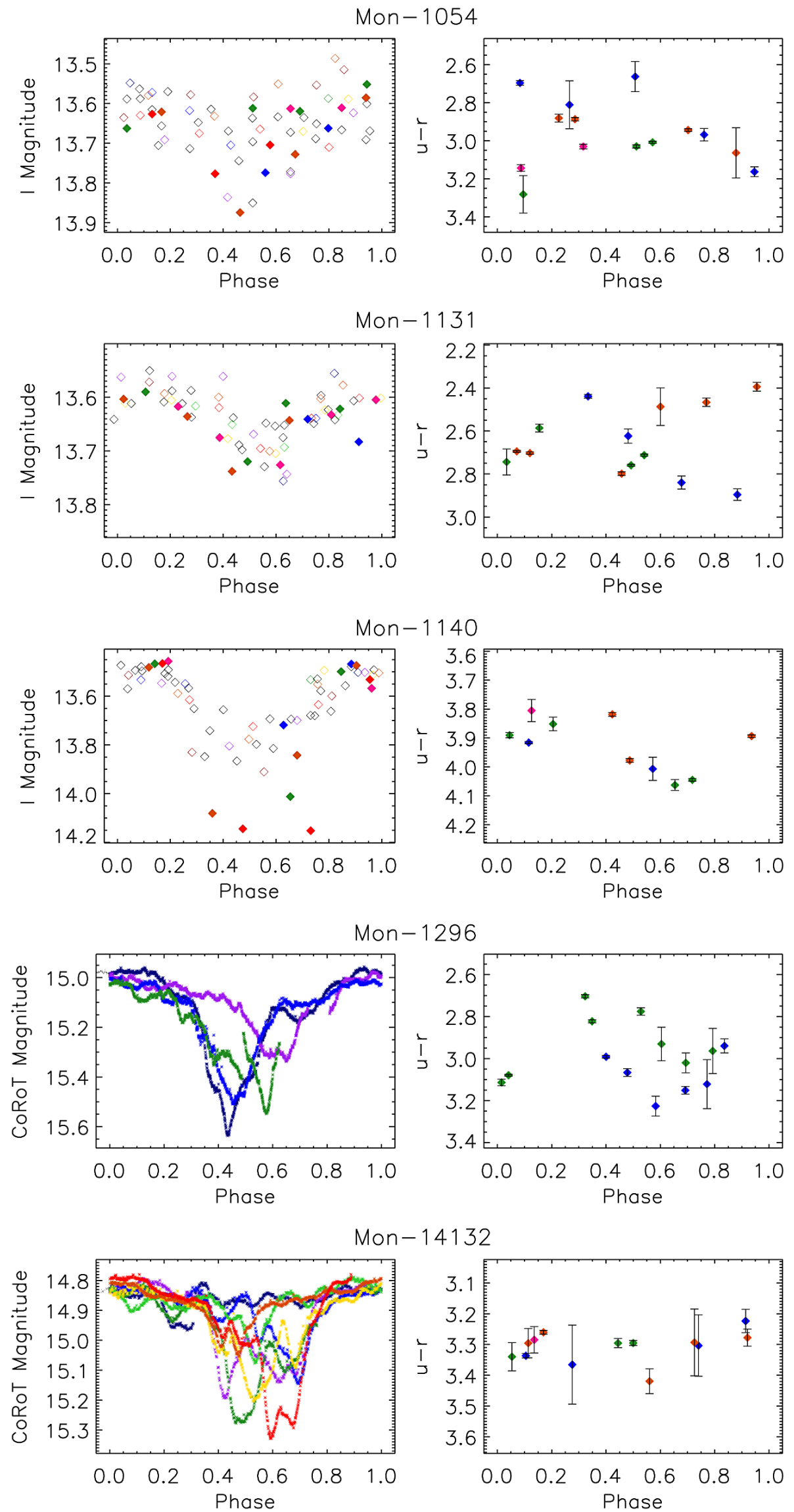

Fig. 24. continued. 